\title{
Transmission and Reflection of Electromagnetic Waves in the Presence of Stratified Media
}

\author{
James R. Wait
}

\begin{abstract}
A general analysis is presented for the electromagnetic response of a plane stratified medium consisting of any number of parallel homogeneous layers. The solution is first developed for plane-wave incidence and then generalized to both cylindrical and sphericalwave incidence. Numerical results for interesting special cases are presented and discussed. The application of the results to surface-wave propagation over a stratified ground is considered in some detail.
\end{abstract}

\section{Introduction}

In the analysis of antenna radiation and kindred problems, it is usually necessary to consider the inhomogeneous nature of the surrounding media. A good example is when an antenna is located over the surface of the earth. In many cases such as this, it is permissible to regard the ground as a semi-infinite medium with a plane surface. Furthermore, this halfspace can often be idealized as a homogeneous medium. As long ago as 1907, Zenneck [1] ${ }^{1}$ showed that a solution of Maxwell's equation could be obtained for such a region by matching certain wave functions at the interface separating the two half-spaces. His solution was characterized as a wave which propagated along the interface without change of waveform with a velocity greater than that of light and was exponentially attenuated with height above the interface (towards the air).

In 1909 Sommerfeld [2] presented an elegant solution for the fields of dipole sources (electric and magnetic) which were located in the air over a homogeneous flat earth. In the final reduction of the integrals to a form suitable for computation, a small but significant error was made. This error was first pointed out explicitly by Norton [3], but not before many had accepted the earlier solution. To compound the confusion, it turned out that Zenneck's surface wave was very similar to Sommerfeld's solution in his 1909 paper. Since that time a large number of papers have appeared treating this subject. The confusion that remains is largely due to the differences in terminology.

The discussion given here relates to three related aspects of this problem. The first is a general analysis of reflection of plane waves from a parallel stratified medium consisting of $M$ homogeneous slabs, the second is the extension to a line source over the stratified medium, and the third is the generalization to dipoles or current elements over the stratified medium. A special case of the latter is when the number $M$ becomes 1 , corresponding to the Sommerfeld problem for a half-space.

\section{Plane-Wave Incidence}

A plane wave with a time factor $\exp (i \omega t)$ is incident at an angle $\theta$ on a stratified medium composed of $M$ homogeneous layers. The electric vector is in the plane of incidence ( $x z$ plane). The situation is illustrated in figure 1 where the $y$ axis is out of the paper. The electrical constants of the layers are $\sigma_{\mathrm{m}}, \boldsymbol{\epsilon}_{\mathrm{m}}$, and $\mu_{\mathrm{m}}$ where the subscript $m$ indicates the $m$ th layer below the surface.

From symmetry it can be seen that the magnetic field has only a $y$ component and for the $m$ th layer, it is a solution of the equation

where

$$
\left(\nabla^{2}-\gamma_{m}^{2}\right) H_{m y}=0,
$$

$$
\gamma_{m}^{2}=i \sigma_{m} \mu_{m} \omega-\epsilon_{m} \mu_{m} \omega^{2}
$$

${ }_{1}^{1}$ Figures in brackets indicate the literature references at the end of this paper. 


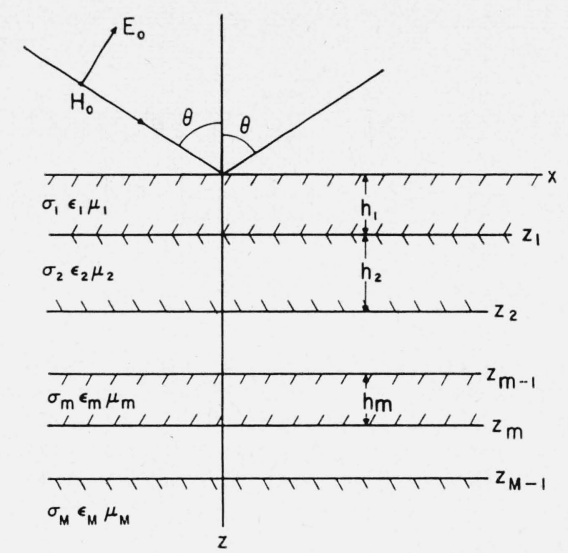

Figure 1. A stratified medium consisting of $M$ homogeneous layers.

The general solution is of the form

where

$$
H_{m y}=\left[a_{m} e^{-u_{m} z}+b_{m} e^{u_{m} z}\right] e^{-i \lambda x},
$$

$$
u_{m}^{2}=\lambda^{2}+\gamma_{m}^{2},
$$

and $\lambda$ can take any value. The incident field $H^{\text {inc. }}$ can be written

$$
H_{0 y}^{\text {inc. }}=H_{0} e^{-\gamma_{0} \cos \theta \cdot z} \times e^{-\gamma_{0} \sin \theta x} .
$$

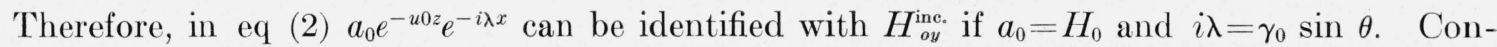
sequently, $b_{0} e^{u_{0} z} e^{-i \lambda x}$ is a reflected wave, and the angle of reflection is $\theta$.

The boundary conditions at the interface $z=0, z=z_{1}, \ldots, z=z_{\mathrm{m}-1}$ are that the tangential fields should be continuous. Now since

$$
E_{m x}=-\left(\sigma_{m}+i \omega \epsilon_{m}\right)^{-1} \frac{\partial H_{m y}}{\partial z}
$$

this means that the boundary conditions can be written

$$
\left[\begin{array}{c}
H_{m-1, y}=H_{m . y} \\
\left(\sigma_{m-1}+i \omega \epsilon_{m-1}\right)^{-1} \frac{\partial H_{m-1, y}}{\partial z}=\left(\sigma_{m}+i \omega \epsilon_{m}\right)^{-1} \frac{\partial H_{m, y}}{\partial z}
\end{array}\right]_{z=z_{m-1}},
$$

where $m=0,1,2, \ldots \quad M-2, M-1$.

Imposing the condition that only outgoing waves are permissible in the lowest layer (which is semi-infinite) it follows that $b_{M}=0$. The boundary conditions then lead to $2(M-1)$ equations that are linear in $a_{m}$ and $b_{m}$ to solve for $2(M-1)$ unknowns in terms of the known coefficient $a_{0}$. The solution ${ }^{2}$ is

$$
\frac{b_{0}}{a_{0}}=\frac{K_{0}-Z_{1}}{K_{0}+Z_{1}}
$$

where

$$
\begin{aligned}
& Z_{1}=K_{1} \frac{Z_{2}+K_{1} \tanh u_{1} h_{1}}{K_{1}+Z_{2} \tanh u_{1} h_{1}} \\
& Z_{2}=K_{2} \frac{Z_{3}+K_{2} \tanh u_{2} h_{2}}{K_{2}+Z_{3} \tanh u_{2} h_{2}}
\end{aligned}
$$

?The quantity $\lambda$ should not be confused with the wavelength. 


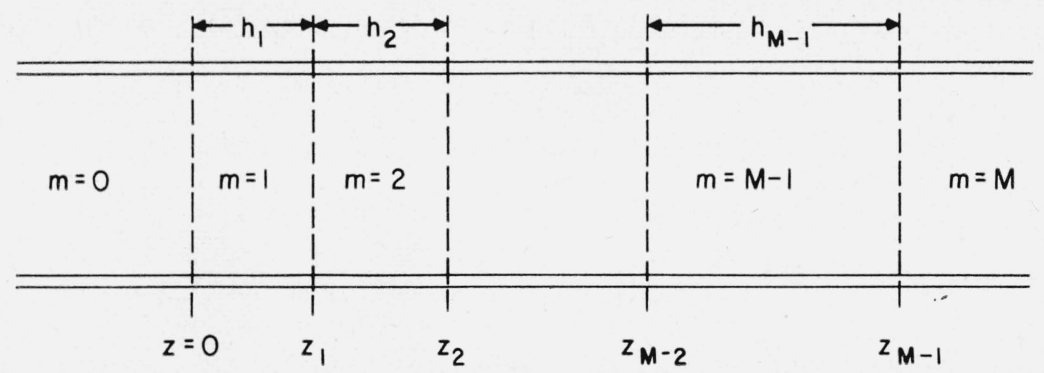

FiguRE 2. Transmission line analogy for the stratified medium of $M$ layers.

$$
\begin{gathered}
Z_{m}=K_{m} \frac{Z_{m+1}+K_{m} \tanh u_{m} h_{m}}{K_{m}+Z_{m+1} \tanh u_{m} h_{m}} \\
Z_{M-1}=K_{M-1} \frac{K_{M}+K_{M-1} \tanh u_{M-1} h_{M-1}}{K_{M-1}+K_{M} \tanh u_{M-1} h_{M-1}},
\end{gathered}
$$

where

$$
K_{m}=\frac{u_{m}}{\sigma+i \omega \epsilon_{m}}, \text { and } u_{m}=\left(\lambda^{2}+\lambda_{m}^{2}\right)^{1 / 2}
$$

(Note that $h_{M}$ is the thickness of the $m$ th slab).

The quantity $b_{0} / a_{0}$ is the ratio of the amplitude of the reflected wave to the amplitude of the incident wave. It is denoted by $R_{\|}$to indicate that the electric field of the incident wave (and also that of the reflected wave) is in the plane of incidence.

The present problem has a well-defined analogy in transmission line theory.

In this analogy each section of the multiple tandemed line is to correspond to a slab. The voltage across the line is $E_{m x}$ and the current is $H_{m y}$ for the $m$ th section. The propagation constant is $u_{m}$ and the characteristic or surge impedance is $K_{m}$ of the $m$ th slab. The incident wave that comes from the left in figure 2 is given by $a_{0} e^{-u_{0} z}$, and the wave reflected at the junction, $z=0$, is $b_{0} e^{u_{0} z}$. The input impedance of the line that is the ratio of the voltage to the current at $z=0$, is $Z_{1}$. Furthermore, the impedance at the junction $z=z_{m}$ is $Z_{m+1}$. With this analogy and a knowledge of the behavior of one-dimensional transmission lines, one could write down the solution of the two-dimensional reflection problem.

Some features of the wave problem will now be discussed. The quantity $Z_{1}$ will play an important role in the following. Since

$$
\left.\left.Z_{1}=E_{0 x} / H_{0 y}\right]_{z=0}=E_{1 x} / H_{1 y}\right]_{z=0},
$$

it is called the surface impedance, being the ratio of the tangential fields at the air-ground interface. In the case of normal incidence, $\theta=0$ and $\lambda=0$, so that $u_{m}=\gamma_{m}$ and $K_{m}=\eta_{m}$, where

and

$$
\gamma_{m}=\left[i \sigma_{m} \mu_{m} \omega-\boldsymbol{\epsilon}_{m} \mu_{m} \omega^{2}\right]^{1 / 2}
$$

$$
\eta_{m}=\left[i \mu_{m} \omega / \sigma_{m}+i \epsilon_{m} \omega\right]^{1 / 2} .
$$

For example, in the case of a homogeneous ground $\left(h_{1} \rightarrow \infty\right)$,

$$
Z_{1}=\eta_{1}, \quad K_{0}=\eta_{0},
$$

and the reflection coefficient becomes simply

$$
R_{\|}=\frac{b_{0}}{a_{0}}=\frac{\eta_{0}-\eta_{1}}{\eta_{0}+\eta_{1}}
$$


Another special case of considerable interest is when $\theta$ approaches $90^{\circ}$ corresponding to glancing incidence, then

$$
u_{m} \cong\left(\gamma_{m}^{2}-\gamma_{0}^{2}\right)^{1 / 2}, \quad K_{m} \cong\left(\gamma_{m}^{2}-\gamma_{0}^{2}\right)^{1 / 2} /\left(\sigma_{m}+i \omega \epsilon_{m}\right),
$$

which yields for the homogeneous ground

$$
Z_{1}=K_{1}=\eta_{1}\left(1-\gamma_{0}^{2} / \gamma_{1}^{2}\right)^{1 / 2}
$$

This special value of $Z_{1}$, which relates the tangential fields in the limiting case of glancing incidence, turns out to be an important quantity in further work. For this reason, it is denoted by $Z^{v}$, where the superscript $v$ indicated that the electric field in the air is nearly vertical for $\left|\gamma_{1}\right|>>\left|\gamma_{0}\right|$. This fact can be shown by evaluating the wave tilt, which is defined by

$$
\left.W=\frac{E_{0 x}}{E_{0 z}}\right]_{z=0}
$$

and is the complex ratio of the horizontal to the vertical electric field in the air just above the ground. It readily follows that for the general case [4]

$$
\left.W=\frac{E_{1 x} / H_{1 y}}{E_{0 z} / H_{0 y}}\right]_{z=0}=\frac{i \omega \epsilon_{0} Z_{1}}{\gamma_{0} \sin \theta}
$$

For a homogeneous ground

$$
W=\frac{i \omega \epsilon_{0} K_{1}}{\gamma_{0} \sin \theta}=\frac{\eta_{1}}{\eta_{0}} \frac{\left(1-\frac{\gamma_{0}^{2}}{\gamma_{1}^{2}} \sin ^{2} \theta\right)^{1 / 2}}{\sin \theta},
$$

which for grazing incidence becomes

$$
W=\frac{\eta_{1}}{\eta_{0}}\left(1-\frac{\gamma_{0}^{2}}{\gamma_{1}^{2}}\right)^{1 / 2}=\left(\frac{\mu_{1}}{\mu_{0}}\right) \frac{\gamma_{0}}{\gamma_{1}}\left(1-\frac{\gamma_{0}^{2}}{\gamma_{1}^{2}}\right)^{1 / 2}
$$

For radio frequencies $\left(\omega \cong 10^{6}\right)$ and moderately conducting ground $\left(\sigma \cong 10^{-2}\right),\left|\gamma_{0} / \gamma_{1}\right|$ is of the order of $10^{-2}$, and thence $W$ is small and hence $\left|E_{0 z}\right|$ is much greater than $\left|E_{0 x}\right|$.

To indicate in a simple way as is possible, the influence of stratification on the reflection of waves from the ground surface, a two-layer case will be considered. This is effected by letting $h_{2} \rightarrow \infty$. Furthermore, it will be assumed that $\left|\gamma_{1} / \gamma_{0}\right|$ and $\left|\gamma_{2} / \gamma_{0}\right|>>1$. Then for any angle of incidence

and

$$
u_{m}=\gamma_{m}\left(1-\frac{\gamma_{0}^{2}}{\gamma_{m}^{2}} \sin ^{2} \theta\right)^{1 / 2} \cong \gamma_{m} \quad(m=1,2),
$$

This leads to the simple relation

$$
K_{m}=\frac{u_{m}}{\sigma_{m}+i \epsilon_{m} \omega} \cong \eta_{m} \quad(m=1,2) .
$$

where

$$
Z_{1} \cong Q K_{1}
$$

$$
Q=\frac{\left(\gamma_{1} / \gamma_{2}\right)+\tanh \gamma_{1} h_{1}}{1+\left(\gamma_{1} / \gamma_{2}\right) \tanh \gamma_{1} h} \text { for } \quad \mu_{1}=\mu_{2}=\mu_{0}
$$

and where $Q$ is the correction to the characteristic impedance $K_{1}$ of the upper layer to account for the presence of the lower layer. Note then, if $\left|\gamma_{1} h_{1}\right|>>1, Q \cong 1$. It can be said that the lower layer is not detectable when $|Q|$ is within 5 percent of unity. Such a condition is met when $\left(\sigma_{1} \mu_{0} \omega\right)^{1 / 2} h>3$.

It should also be noted that $?$ relates the wave tilts for a stratified (two-layer) ground and that of a homogeneous ground by $W \cong W_{0}$ ?, where 


$$
\left.W_{0} \cong W\right]_{h_{1}=\infty} .
$$

An example is here quoted to illustrate the order of magnitude of the quantities involved:

Frequency, $f=\omega / 2 \pi=125 \mathrm{kc}$

Upper layer conductivity, $\sigma_{1}=10^{-2} \mathrm{mho} / \mathrm{m}$,

Dielectric constant of air, $\epsilon_{0}=8.854 \times 10^{-12} \mathrm{f} / \mathrm{m}$,

Dielectric constant of ground, $\epsilon_{1}=10 \cdot \epsilon_{0}$,

Magnetic permeability, $\mu=4 \pi \times 10^{-7} \mathrm{~h} / \mathrm{m}$.

For these values, $W_{0}=0.082 / 41.1^{\circ}$, for a homogeneous ground. In the case of a two-layer ground where $h_{1}$ is finite,

$$
\begin{aligned}
W & =W_{0} Q \\
& =0.082|Q| / \underline{41.1^{\circ}+q},
\end{aligned}
$$

where $q$ is the argument of $Q$ expressed in degrees. For frequencies of this order, $\epsilon_{1} \omega / \sigma_{1}$ and $\epsilon_{2} \omega / \sigma_{2}$ are small (in the above example $\left.\epsilon_{1} \omega / \sigma_{1}=0.0069\right)$. Therefore, $\gamma_{1} \cong\left(i \sigma_{1} \mu \omega\right)^{1 / 2}$ and $\gamma_{2} \simeq$ $\left(i \sigma_{2} \mu \omega\right)^{1 / 2}$. A formula suitable for computation of $Q$ is then given by

$$
\begin{aligned}
Q & =\frac{\left(\sigma_{1} / \sigma_{2}\right)^{1 / 2}+\tanh \sqrt{i} V}{1+\left(\sigma_{1} / \sigma_{2}\right)^{1 / 2} \tanh \sqrt{i} V} \\
& =\tanh \left[\sqrt{i} V+\tanh ^{-1}\left(\sigma_{1} / \sigma_{2}\right)^{1 / 2}\right] .
\end{aligned}
$$

If $\sigma_{2}>>\sigma_{1}$ corresponding to a highly conducting substratum,

$$
Q \simeq \tanh \sqrt{i} V
$$

or if $\sigma_{2}<<\sigma_{1}$ corresponding to an insulating substratum,

$$
Q \cong \operatorname{coth} \sqrt{i} V \text {. }
$$

In the above example, the parameter $V$ can be replaced by $h_{1} / 10$, where $h_{1}$ is the thickness of the upper stratum in meters. The function $Q$ and its argument $q$ are plotted in figure 3a and $3 \mathrm{~b}$ as a function of $V$ for various values of the ratio $\sigma_{2} / \sigma_{1}$.
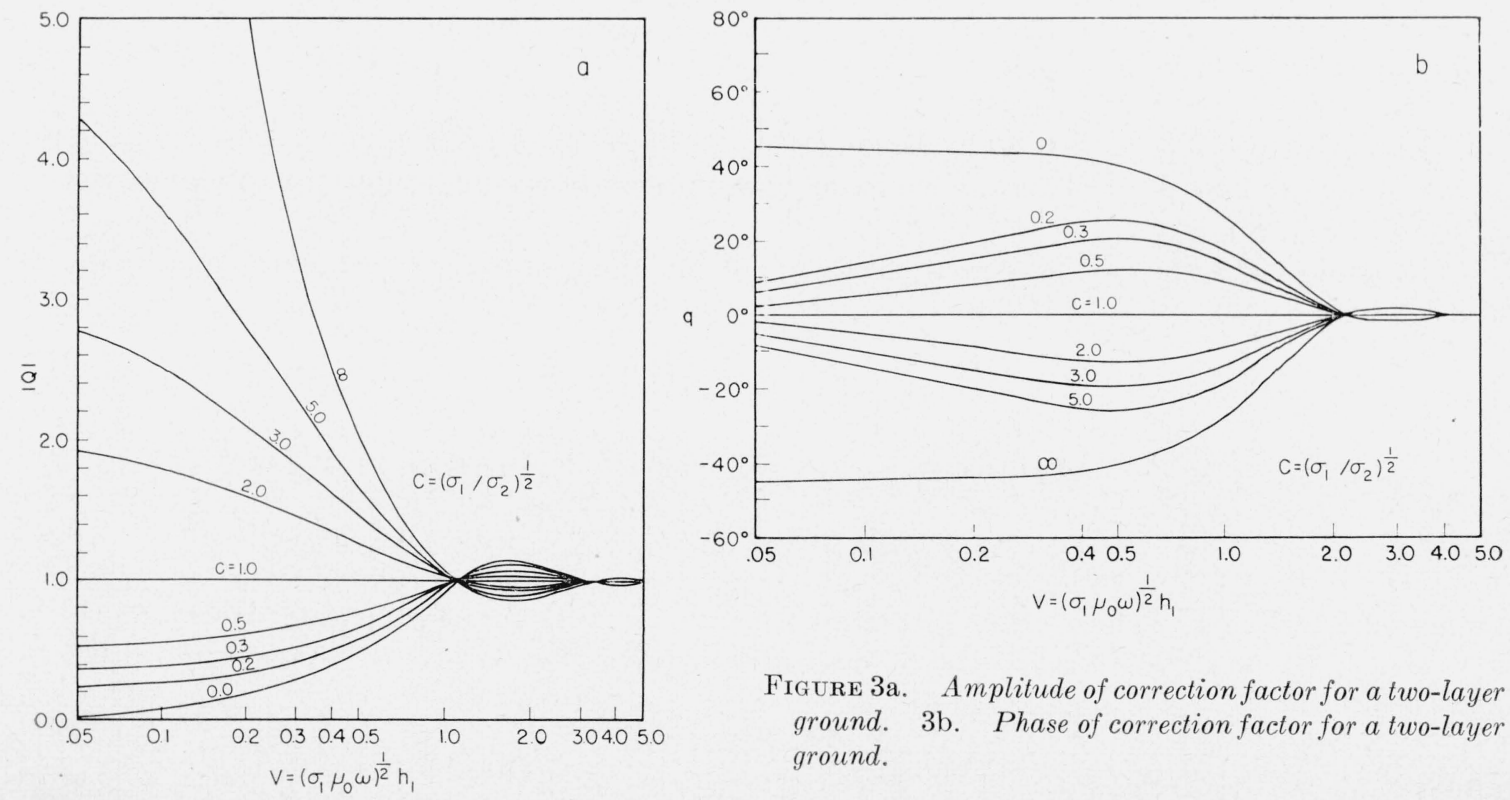

Figure 3a. Amplitude of correction factor for a two-layer ground. $3 \mathrm{~b}$. Phase of correction factor for a two-layer ground. 


\section{Extension to Perpendicular Incidence}

In the preceding problem, the incident-plane wave has the electric vector contained in the plane of incidence (and the magnetic vector parallel to the interfaces). For this reason, it is termed "parallel incidence". The other important case is when the electric vector is perpendicular to the plane of incidence. This is termed "perpendicular incidence".

Again choosing the plane of incidence to be the $(x z)$ plane, the incident wave now has only a $y$ component of the electric field. By analogy to eq 2, the general solution is of the form

$$
E_{m y}=\left[\bar{a}_{m} e^{-u_{m} z}+\bar{b}_{m} e^{u_{m} z}\right] e^{-i \lambda x},
$$

where $u_{m}^{2}=\lambda^{2}+\gamma_{m}^{2}, \quad \bar{a}_{0}$ is the amplitude of the incident wave, $\bar{b}_{0}$ is the amplitude of the reflected wave, and $\bar{b}_{M}=0$. In this case,

$$
i \mu_{m} \omega H_{m x}=\frac{\partial E_{m y}}{\partial z}
$$

so that the boundary conditions now become

$$
\begin{gathered}
E_{m-1, y}=E_{m, y} \\
\left.\left(i \mu_{m-1} \omega\right)^{-1} \frac{\partial E_{m-1, y}}{\partial z}=\left(i \mu_{m} \omega\right)^{-1} \frac{\partial E_{m, y}}{\partial z}\right]_{z=z_{m}} .
\end{gathered}
$$

These are transformable to the boundary conditions for parallel incidence by making the substitutions: $E_{m y}$ for $H_{m y}, i \mu_{m} \omega$ for $\sigma_{m}+i_{\epsilon_{m}} \omega$. Then using the previous results, the solution for perpendicular incidence can be written down:

where

$$
\frac{\bar{b}_{0}}{\bar{a}_{0}}=\frac{N_{0}-Y_{1}}{N_{0}+Y_{1}}
$$

for $m=1,2,3, \ldots, M-1$ and

$$
Y_{m}=N_{m} \frac{Y_{m+1}+N_{m} \tanh u_{m} h_{m}}{N_{m}+Y_{m+1} \tanh u_{m} h_{m}}
$$

In the preceding

$$
Y_{M}=N_{M}
$$

$$
N_{m}=\frac{u_{m}}{i \mu_{m} \omega}
$$

where, as before, $u_{m}=\left(\lambda^{2}+\gamma_{m}^{2}\right)^{1 / 2}$.

The quantity $\bar{b}_{0} / \bar{a}_{0}$, which is the ratio of the amplitude of the reflected wave to the incident wave, is denoted by $R_{\perp}$. There is a similar transmission line analogy for this problem, which need not be pointed out.

In analogy to the surface-impedance function $Z_{1}$, the quantity $Y_{1}$ is a surface admittance and is given by

$$
\left.\left.Y_{1}=-H_{0 x} / E_{0 y}\right]_{z=0}=-H_{1 x} / E_{1 y}\right]_{z=0}
$$

In the case of a homogeneous ground at glancing incidence $(\theta \rightarrow \pi / 2)$, it follows that

$$
Y_{1}=N_{1}=\left(1 / \eta_{1}\right)\left(1-\gamma_{0}^{2} / \gamma_{1}^{2}\right)^{1 / 2},
$$

which is denoted $Y^{h}$, where the superscript $h$ indicates that the electric field is horizontal in contrast to the near vertical electric field associated with $Z^{v}$. It is interesting to note that

and for $\left|\gamma_{1}^{2}\right|>>\left|\gamma_{0}^{2}\right|, Y^{h} Z^{v} \cong 1$.

$$
Y^{h} Z^{v}=\left(1-\gamma_{0}^{2} / \gamma_{1}^{2}\right)
$$




\section{Impedance Matching and Natural Oscillations in Stratified Media}

In this section, some remarks will be made concerning the nature of extreme conditions where the reflection coefficient on a plane stratified media becomes zero or infinite. The discussion will be confined primarily to parallel incidence, although the results are easily carried over to perpendicular incidence.

As indicated, the reflection coefficient $R_{\|}$is given by

$$
R_{\|}=\frac{K_{0}-Z_{1}}{K_{0}+Z_{1}}
$$

where

$$
K_{0}=u_{0} / i \omega \epsilon_{0}=\left(\lambda^{2}-k_{0}^{2}\right)^{1 / 2} / i \omega \epsilon_{0}
$$

and $Z_{1}$ is the normal impedance at the interface $z=0$. In the case of a homogeneous half space $\left(h_{1} \rightarrow \infty\right)$,

$$
Z_{1}=K_{1}=u_{1} /\left(\sigma_{1}+i \omega \epsilon_{1}\right)=\left(\lambda^{2}+\gamma_{1}^{2}\right)^{1 / 2} /\left(\sigma_{1}+i \omega \epsilon_{1}\right)
$$

The condition for matching is that $R_{\Downarrow}=0$ corresponding to the absence of a reflected wave. This requires that

$$
K_{0}=Z_{1}
$$

For a homogeneous half-space, the condition is simply

which, when solved for $\lambda$, yields

$$
K_{0}=K_{1}
$$

$$
\lambda= \pm\left[\frac{\mu_{1}^{2} / \gamma_{1}^{2}-\mu_{0}^{2} / \gamma_{0}^{2}}{\mu_{0}^{2} / \gamma_{0}^{4}-\mu_{1}^{2} / \gamma_{1}^{4}}\right]
$$

In the case of no magnetic permeability contrast $\left(\mu_{1}=\mu_{0}\right)$, the above simplifies ${ }^{3}$ to

$$
i \lambda= \pm \frac{\gamma_{1} \gamma_{0}}{\left(\gamma_{1}^{2}+\gamma_{0}^{2}\right)^{1 / 2}}
$$

and if both media are perfect dielectrics $\gamma_{1}=i k_{1}$ and $\gamma_{0}=i k_{0}$,

$$
\lambda= \pm \frac{k_{1} k_{0}}{\left(k_{1}^{2}+k_{0}^{2}\right)^{1 / 2}} .
$$

But, since $\lambda=k_{0} \sin \theta$, the preceding equations for a condition of matching can be employed to determine the angle of incidence when there shall be no reflection. In the case of the two dielectric half-spaces, the condition is

$$
\sin \theta= \pm \frac{k_{1}}{\left(k_{1}^{2}+k_{0}^{2}\right)^{1 / 2}}
$$

or $\tan \theta= \pm\left(k_{1} / k_{0}\right)$. This latter equation is well known from optics, and there the angle $\theta$ is known as the Brewster angle, and the ratio $k_{1} / k_{0}$ is called the relative refractive index.

It is indeed very interesting to observe that if $k_{1}$ is not real, (or $\gamma_{1}$ not imaginary), the quantity $\lambda$ or $\theta$ required for matching is complex. That is, in the language of optics, the Brewster angle for an absorptive homogeneous medium is complex. The existence of plane waves with a complex angle of incidence will not be discussed at this stage, but it is interesting to note that the so-called Zenneck surface wave is very similar to the disturbance propagating along the interface, when there is a condition of wave matching. His solution is briefly described below.

${ }^{3}$ In this case the wave tilt $W=\gamma_{0} / \gamma_{1}$, as can be deduced from eq. 11. 
Zenneck [1] postulated that a surface wave could propagate along the interface $z=0$ between two homogeneous media. He assumed that in the upper medium $(z<0)$

and in the lower medium

$$
H_{0 y}=b_{0} e^{+u_{0} z} e^{-i \lambda x},
$$

$$
H_{1 y}=a_{1} e^{-u_{1} z} e^{-i \lambda x},
$$

for propagation in the $x$-direction. The signs prefixing $u_{0}$ and $u_{1}$ were chosen to insure that the field decayed to zero for $|z| \rightarrow \infty$. The corresponding electric fields are then obtained from Maxwell's equations,

and

$$
E_{0 y}=-K_{0} b_{0} e^{u_{0} z} e^{-i \lambda x}
$$

$$
E_{1 y}=K_{1} a_{1} e^{-u_{1} z} e^{-i \lambda x} .
$$

The boundary conditions then require that $b_{0}=a_{1}$, and

$$
K_{0}+K_{1}=0 \quad \text { or } \frac{u_{0}}{\epsilon_{0}}+\frac{u_{1}}{\epsilon_{1}-i \sigma_{1} / \omega}=0
$$

The latter condition cannot be met for any value of $\lambda$ if the imaginary parts of $u_{0}$ and $u_{1}$ are to be greater than zero. It now appears that Zenneck solved the matching equation $K_{0}=K_{1}$ in place of $K_{0}=-K_{1}$.

Returning to the general case, it is seen that

$$
R_{\|}=\frac{K_{0}-Z_{1}}{K_{0}+Z_{1}}
$$

has a pole (i. e., becomes infinite) when

$$
K_{0}+Z_{1}=0
$$

This is termed the "resonance condition." For the homogeneous half-space, this resonance condition is simply $K_{0}+K_{1}=0$, which is identical to Zenneck's equation for a surface wave.

Although it is not possible to set up a surface wave in the sense envisaged by Zenneck, it is possible to retain his solutions if the disturbance in the upper medium is a wave containing: a factor $e^{-u_{0} z}$ in place of $e^{+u_{0} z}$, and the boundary condition becomes $K_{0}=K_{1}$, which corresponds to the "matching" condition.

In the case of a stratified media, it may well happen that the "resonance" condition $K_{0}+Z_{1}=0$ is satisfied. Rewriting this as

$$
Z_{1}=i \frac{u_{0}}{\epsilon_{0} \omega}
$$

and since for a surface wave $u_{0}$ is to be mainly real (i. e., small imaginary component), then $Z_{1}$ is to be mainly imaginary (in the positive sense). Such a surface characteristic is described as inductive. The quantity $\lambda$, which describes the transverse propagation, is mainly real and somewhat greater than $k_{0}$ [i.e., $\lambda^{2}=u_{0}^{2}+k_{0}^{2}$ ].

A simple example of an inductive surface is a highly conducting plane, coated with a thin uniform film of dielectric. From eq 6 with $Z_{2}=0$ (or $\gamma_{2}=\infty$ )

$$
Z_{1} \cong K_{1} \tanh u_{1} h_{1}
$$

where $h_{1}$ is the thickness of the film. For thin films, and $\mu_{0}=\mu_{1}$,

$$
Z_{1} \cong K_{1} u_{1} h_{1}=i \mu_{0} \omega h_{1}\left[1-\frac{\lambda^{2}}{k_{1}^{2}}\right] .
$$

This can be further approximated to

$$
Z_{1}=i \mu_{0} \omega h_{1}\left[1-\left(k_{0} / k_{1}\right)^{2}\right],
$$


since $\lambda$ is near $k_{0}$. Such a surface is therefore almost purely inductive. In this case,

$$
u_{o}=\epsilon_{0} \mu_{0} \omega^{2} \bar{h}=k_{0}^{2} \bar{h},
$$

and

$$
\lambda=\left(u_{0}^{2}+k_{0}^{2}\right)^{1 / 2}=k_{0}\left[1+\left(k_{0} \bar{h}\right)^{2}\right]^{1 / 2} \cong k_{0}\left[1+\left(k_{0} \bar{h}\right)^{2} / 2\right],
$$

where $\bar{h}=h_{1}\left(1-k_{0}^{2} / k_{1}^{2}\right)$. The wave has a phase velocity in the transverse $(x)$ direction, which is $1-\left(k_{0} h\right)^{2} / 2$ times that of free space, and it decays rapidly in the $z$-direction. This approximate solution is valid if $k_{0} \bar{h}$ (or $k_{0} h_{1}$ ) is small compared to unity.

In general, the determination of the poles in the reflection coefficient $R_{\|}$leads to transcendental equations that must be solved by numerical or graphical means. A discussion of surface waves on dielectric coated conductors without the restriction that $h_{1}$ be small has been given by Atwood [5a]. The subject has also been discussed by Goubau [5b].

Further remarks regarding surface waves will be made in a later section following the analysis of the excitation problem.

\section{Line-Source Excitation}

In problems of reflection from stratified media, it is more meaningful from a physical standpoint to consider the source a localized distribution of current. No physical source in existence produces pure plane waves, although in certain limiting cases the fields have characteristics closely akin to plane waves. An example is a line source of electric current. It was shown that at large distances from the source, the electric and magnetic field were mutually orthogonal to one another and to the direction of propagation [7]. It might then be conjectured that the reflected wave for a line source located high over a plane interface could be computed from the plane-wave reflection coefficient. Such is the case if certain limitations are imposed.

It is the purpose of this section to extend the plane-wave solution to the case of an (infinite) line source of (constant) electric current located at height $h$ above the stratified medium considered in the previous section. The coordinate of the line source is taken as $(0, y,-h)$, as indicated in figure 4.

In view of the symmetry, the electric field only has a $y$ component $E_{m}$ for any of the layers (the subscript $y$ on $E_{m}$ is dropped for convenience). The direct, or primary, field $E_{0}^{p}$ in the region $z<0$ (above the interface) is expressible as

$$
E_{0}^{p}=-\frac{i \mu \omega I}{2 \pi} K_{0}\left[\gamma_{0}\left[x^{2}+(z+h)^{2}\right]^{1 / 2}\right],
$$

where $K_{0}$ is a modified Bessel function. To convert the expression for $E_{0}^{p}$ to a more useful

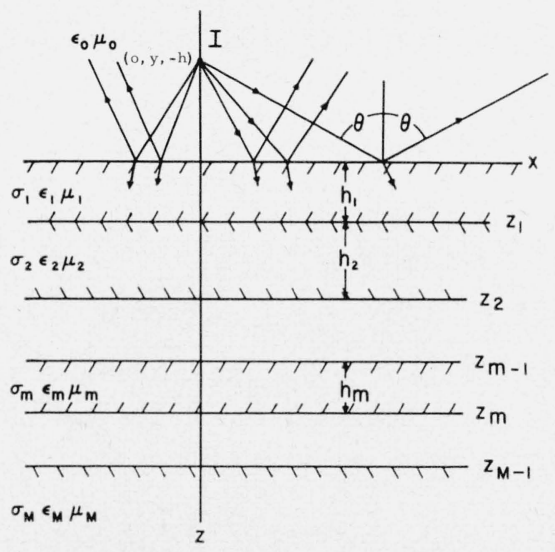

Figure 4. Line source over a layered half-space. 
form, it is written as a Fourier integral as follows:

$$
E_{0}^{p}=\int_{-\infty}^{\infty} A(\lambda) e^{-i \lambda x} d \lambda
$$

By making use of the inverse property of the Fourier integral, it follows that

$$
\begin{aligned}
A(\lambda) & =\frac{1}{2 \pi} \int_{-\infty}^{\infty} E_{0}^{p} e^{i \lambda x} d x \\
& =\frac{-i \mu_{0} \omega I}{4 \pi^{2}} \int_{-\infty}^{+\infty} K_{0}\left[\gamma_{0}\left[x^{2}+(z+h)^{2}\right]^{1 / 2}\right] e^{i \lambda x} d x .
\end{aligned}
$$

This integral is evaluated to give ${ }^{4}$

$$
A(\lambda)=-\frac{i \mu_{0} \omega I}{2 \pi u_{0}} e^{ \pm u_{0}(z+h)}
$$

where $u_{0}=\left(\lambda^{2}+\gamma_{0}^{2}\right)^{1 / 2}$ and where the $+\operatorname{sign}$ is to be employed for $(z+h)<0$ and the $-\operatorname{sign}$ for $(z+h)>0$.

The resultant field in the space $0>z>-h$ is then written in the form

$$
E_{0}=\frac{-i \mu_{0} \omega I}{4 \pi} \int_{-\infty}^{\infty} u_{0}^{-1}\left[e^{-u_{0}(z+h)}+R_{\perp}(\lambda) e^{u_{0}(x-h)}\right] \exp (-i \lambda x) d \lambda,
$$

where the term containing $R_{\perp}(\lambda)$ accounts for the presence of the stratified medium at $z=0$ and is as yet unknown. $\mathrm{Eq}(48)$ satisfies the equation $\left(\nabla^{2}-\gamma_{0}^{2}\right) E_{0}=0$, and behaves in the proper manner as $z \rightarrow-h$ and $x \rightarrow 0$. The corresponding expression for $E_{0}$ in the region $-h>z>-\infty$ is identical to eq (48), except that the (-) sign on the first exponential should be changed to a + sign. $E_{0}$ then decays properly to zero as $z \rightarrow-\infty$.

The integral representation for $E_{0}$ has a clear physical meaning. When the symbol $\lambda$ is identified with $k_{0} \sin \theta$ or $-i \gamma_{0} \sin \theta$, the field $E_{0}^{p}$ is a spectrum of plane waves of (complex) angle $\theta$ of incidence. The complete field $E_{0}$ contains a spectrum of both incident and reflected waves [7]. The structure of the integrand in the integral representation for $E_{0}$ is identical to the corresponding factor in the plane-wave solution in the previous section. In fact, the correspondence carries over to each of the sublayers. For example, in the present problem, for $m=0$ to $M$,

and

$$
E_{m}=\int_{-\infty}^{\infty}\left[\bar{a}_{m}(\lambda) e^{-u_{m} z}+b_{m}(\lambda) e^{u_{m} z}\right] e^{-i \lambda x} d \lambda
$$

$$
i \mu_{m} \omega H_{m x}=\frac{\partial E_{m}}{\partial z}, \quad-i \mu_{m} \omega H_{m z}=\frac{\partial E_{m}}{\partial x} \text {. }
$$

The solution of the present problem ${ }^{5}$ is thus immediate;

where

$$
R_{\perp}(\lambda)=\frac{\bar{b}_{0}(\lambda)}{\bar{a}_{0}(\lambda)}=\frac{N_{0}(\lambda)-Y_{1}(\lambda)}{N_{0}(\lambda)+Y_{1}(\lambda)}
$$

$$
Y_{m}(\lambda)=\frac{Y_{m+1}(\lambda)+N_{m}(\lambda) \tanh u_{m} h_{m}}{N_{m}(\lambda)+Y_{m+1}(\lambda) \tanh u_{m} h_{m}}
$$

with $m=1,2 \ldots, M-1$, and $Y_{M}(\lambda)=N_{M}(\lambda)$.

Equation (48), along with (50) and (51), constitutes the formal solution of the problem. The remaining task is the evaluation of the integral in (48). Except in certain limiting cases, certain approximations must be made in order to obtain a useful result.

${ }^{4}$ Campbell and Foster [6], pair No. 917.

${ }^{5}$ It should be noted that eq (50) has the same form as eq (21). 
The simplest limiting case is when the interface becomes highly conducting. Then, since $\gamma_{1} \cong \infty, R_{\perp}(\lambda) \cong-1$, and

$$
E_{0}=\frac{-i \mu_{0} \omega I}{4 \pi} \int_{-\infty}^{+\infty} u_{0}^{-1}\left[e^{-u_{0}(z+h)}-e^{u_{0}(z-h)}\right] e^{-i \lambda x} d \lambda
$$

Both these integrals are of the same type and may be readily evaluated in view of eq (46) and (47). Consequently, for $z<0$,

$$
E_{0}=\frac{-i \mu_{0} \omega I}{2 \pi}\left\{K_{0}\left[\gamma_{0}\left[x^{2}+(z+h)^{2}\right]^{1 / 2}\right]-K_{0}\left[\gamma_{0}\left[x^{2}+(z-h)^{2}\right]^{1 / 2}\right]\right\} .
$$

It can be readily verified that $\left(\nabla^{2}-\gamma_{0}^{2}\right) E_{0}=0$ for $-z>0$ and, moreover, $E_{0}=0$ for $z=0$, which is the required boundary condition for a perfect conductor. It should be mentioned that an equally simple result is obtained for cases where $\mu_{1} \cong \infty$, corresponding to a rather hypothetical situation where the interface at $z=0$ is behaving as a perfect magnetic conductor. Then $R_{\perp}(\lambda) \cong+1$ and the solution for $E_{0}$ is identical to eq (53), with the second term inside the braces changing sign.

When $\left|\gamma_{0} h\right|>>1$ and $h>>-z$, eq (53) for $E_{0}$ can be written in the form ${ }^{6}$

$$
E_{0} \cong \frac{-i \mu_{0} \omega I}{2 \pi}\left(\frac{\pi}{2}\right)^{1 / 2} \frac{e^{-\gamma_{0} R_{0}}}{\left(\gamma_{0} R_{0}\right)^{1 / 2}}\left[e^{-\bar{u}_{0}(z)}-e^{+\bar{u}_{0}(z)}\right] e^{-i \bar{\lambda} \bar{x}},
$$

where $\bar{u}_{0}=\left((\bar{\lambda})^{2}+\gamma_{0}^{2}\right)^{1 / 2}, \quad \bar{\lambda}=-i \gamma_{0} \sin \bar{\theta}$, and where $Q$ is the geometrical reflection point such that the ray $I Q$ and the ray $Q P$ make equal angles $\bar{\theta}$ with the normal at $Q$. The quantity $R_{0}$ is the length of the ray from the line source to the point of reflection $Q$. In fact, $Q$ is taken as the new origin of the cartesian system $\bar{x}, y, z$, as indicated in figure 5 .

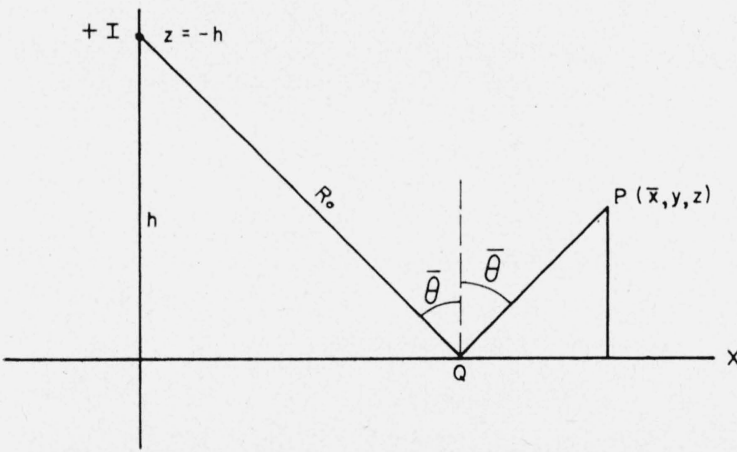

Figure 5. Geometrical interpretation for the secondary field.

Equation (54) can be simplified even further by noting that the quantity prefixing the square bracket is simply the amplitude of the incident wave. When this is normalized to unity,

$$
E_{0}=\left[e^{-\bar{u}_{0}(z)}-e^{+\bar{u}_{0}(z)}\right] e^{-i \overline{\lambda x}},
$$

which is readily identified as an incident and reflected plane wave with an angle of incidence and reflection of $\bar{\theta}$. To generalize, eq (55) for a stratified medium, the reflected wave would be simply modified by a reflection coefficient for an angle of incidence $\bar{\theta}$. The modified form would be

$$
E_{0}=\left[e^{-\bar{u}_{0} z}+R_{\perp}(\bar{\lambda}) e^{+\bar{u}_{0} z}\right] e^{-i \tilde{\lambda} x} .
$$

A suggested modified version of eq (53) to account for the stratified nature of the medium would then be

$$
E_{0} \cong \frac{-i \mu_{0} \omega I}{2 \pi}\left\{K_{0}\left[\gamma_{0}\left[x^{2}+(z+h)^{2}\right]^{1 / 2}\right]+R_{\perp}(\bar{\theta}) K_{0}\left[\gamma_{0}\left[x^{2}+(z-h)^{2}\right]^{1 / 2}\right]\right\}
$$

when $R_{\perp}(\bar{\theta})$ is the reflection coefficient for a plane-wave incident at an angle $\bar{\theta}$ on the interface $z=0$. The angle $\bar{\theta}$ is determined by simple geometrical considerations, as indicated above. ${ }^{7}$

- The first term of the asymptotic expansion of the modified Bessel function $K_{0}(\alpha)$ is only retained, i. e., $K_{0}(\alpha) \cong \epsilon^{-\alpha}(\pi / 2 \alpha)^{1 / 2}$.

- Note then $\tan \bar{\theta}=x /(h-z)$. 
This result, which has been derived by an elementary approach, considers only the specular component. For smaller values of $|z|$ and $h$ the situation becomes more complicated because surface waves within the layers may be excited. This question will not be pursued further at the moment.

\section{Line Source on a Homogeneous Medium}

When the line source is situated at the interface $(h=0)$ between two media of semiinfinite extent with electrical properties $\epsilon_{0}, \mu_{0}$ and $\sigma_{1}, \epsilon_{1}, \mu_{0}$, respectively, the electric field $E_{0}$ is given by

$$
E_{0}=\frac{-i \mu_{0} \omega I}{\pi} \int_{0}^{\infty} \frac{\cos \lambda x}{u_{1}+u_{0}} e^{+u_{0} z} d \lambda,
$$

for $z>0$. This is a special case of eq (48) with $h=0$ and $z_{1}=h_{1}=\infty$. Noting that

it follows that, for $z=0$

$$
\frac{1}{u_{1}+u_{0}}=\frac{u_{1}-u_{0}}{u_{1}^{2}-u_{0}^{2}}=\frac{u_{1}-u_{0}}{\gamma_{1}^{2}-\gamma_{0}^{2}}
$$

Since

$$
E_{0}=\frac{-i \mu_{0} \omega I}{\pi\left(\gamma_{1}^{2}-\gamma_{0}^{2}\right)}\left\{\int_{0}^{\infty} u_{1} \cos \lambda x d \lambda-\int_{0}^{\infty} u_{0} \cos \lambda x d \lambda\right\} .
$$

$$
\operatorname{Lim}_{z \rightarrow 0} \int_{0}^{\infty} \cos \lambda x \frac{e^{-u_{0}|z|}}{u_{0}} d \lambda=K_{0}\left(\gamma_{0} x\right)
$$

which is equivalent to eq $(45)$, it is seen that ${ }^{8}$

$$
\int_{0}^{\infty}(\cos \lambda x) u_{0} d \lambda=\left(\gamma_{0}^{2}-\frac{\partial^{2}}{\partial x^{2}}\right) K_{0}\left(\gamma_{0} x\right) \gamma_{0} x=-K_{1}\left(\gamma_{0} x\right) \gamma_{0} x^{-1}
$$

Making use of this result, the expression for the electric field becomes

$$
E_{0}=\frac{-i \mu_{0} \omega I}{\pi\left(\gamma_{1}^{2}-\gamma_{0}^{2}\right) x^{2}}\left[\gamma_{0} x K_{1}\left(\gamma_{0} x\right)-\gamma_{1} x K_{1}\left(\gamma_{1} x\right)\right]
$$

This formula is exact.

At large values of the argument

$$
K_{n}\left(\gamma_{1} x\right) \cong\left(\frac{\pi}{2 \gamma_{1} x}\right)^{1 / 2} e^{-\gamma_{1} x}
$$

and if the real part of $\gamma_{1} x$ is $>>1$,

$$
E_{0} \cong \frac{-i \mu_{0} \omega I \gamma_{0}}{\pi\left(\gamma_{1}^{2}-\gamma_{0}^{2}\right) x} K_{1}\left(\gamma_{0} x\right)
$$

Furthermore, if $\gamma_{0}=i k_{0}$ where $k_{0} x$ is a real number $>>1$, it follows that

$$
\begin{aligned}
E_{0} & \simeq \frac{-i \mu_{0} \omega I \gamma_{0}}{\pi\left(\gamma_{1}^{2}-\gamma_{0}^{2}\right) x}\left(\frac{\pi}{2 \gamma_{0} x}\right)^{1 / 2} e^{-\gamma_{0} x} \\
& \simeq \frac{\mu_{0} \omega k_{0} I}{\pi\left(\gamma_{1}^{2}+k_{0}^{2}\right)}\left(\frac{\pi}{2 k_{0} x^{3}}\right)^{1 / 2} e^{-i\left(k_{0} x+\pi / 4\right)} .
\end{aligned}
$$

It is convenient to express this in terms of the primary field,

$$
E_{o}^{p}=\frac{-i \mu_{0} \omega I}{2 \pi} K_{0}\left(\gamma_{0} \rho\right) \cong \frac{-i \mu_{0} \omega I}{2 \pi}\left(\frac{\pi}{2 \gamma_{0} x}\right)^{1 / 2} e^{-\gamma_{0} x}
$$

${ }^{8}$ In eq (61) use is made of $d K_{1} / d \alpha=-K_{1} / \alpha-K_{0}$, where $\alpha$ is the argument $\gamma_{0} x$. 
in the following manner:

$$
\frac{E_{0}}{E_{o}^{p}} \cong \frac{2 \gamma_{0}}{\left(\gamma_{1}^{2}-\gamma_{0}^{2}\right) x}=\frac{2 i k_{0}}{\left(\gamma_{1}^{2}+k_{0}^{2}\right) x}
$$

The vertical electric field in the interface, for the current line source in the interface, is given by

$$
\begin{aligned}
H_{0 z} & =H_{1 z}=-\frac{1}{i \mu_{0} \omega} \frac{\partial E_{0}}{\partial x} \\
& =\frac{1}{\pi\left(\gamma_{1}^{2}-\gamma_{0}^{2}\right) x^{3}}\left[2 \gamma_{0} x K_{1}\left(\gamma_{0} x\right)+\gamma_{0}^{2} x^{2} K_{0}\left(\gamma_{0} x\right)-2 \gamma_{1} x K_{1}\left(\gamma_{1} x\right)-\gamma_{1}^{2} x^{2} K_{0}\left(\gamma_{1} x\right)\right]
\end{aligned}
$$

and, if the real part of $\gamma_{1} x>>1$,

$$
H_{0 z} \simeq \frac{\gamma_{0} I}{\pi\left(\gamma_{1}^{2}-\gamma_{0}^{2}\right) x^{2}}\left[2 K_{1}\left(\gamma_{0} x\right)+\gamma_{0} x K_{0}\left(\gamma_{0} x\right)\right],
$$

which simplifies further, for the case $\left|\gamma_{0} x\right|>>1$, to

$$
H_{0 z} \cong \frac{\gamma_{0}^{2} I}{\pi\left(\gamma_{1}^{2}-\gamma_{0}^{2}\right) x}\left(\frac{\pi}{2 \gamma_{0} x}\right)^{1 / 2} e^{-\gamma_{0} x} \cong E_{0} / \eta_{0},
$$

where

$$
\eta_{0}=i \mu_{0} \omega / \gamma_{0}=\mu_{0} \omega / k_{0}=\left(\mu_{0} / \epsilon_{0}\right)^{1 / 2}
$$

The field at very low frequencies near the line source is also of interest. For example if $\left|\gamma_{0} x\right|<<1$,

$$
H_{0 z} \simeq \frac{I}{\pi \gamma_{1}^{2} x^{3}}\left[2-2 \gamma_{1} x K_{1}\left(\gamma_{1} x\right)-\left(\gamma_{1} x\right)^{2} K_{0}\left(\gamma_{1} x\right)\right]
$$

which approaches $-I / 2 \pi x$ as $\gamma_{1} x$ approaches zero.

The magnetic field $H_{0 z}$ expressed as a ratio to the primary field $H_{0 z}^{p}(=-I / 2 \pi x)$ is plotted in figure 6 as a function of $\left|\gamma_{1} x\right|$ under the assumption that $\epsilon_{1} \omega / \sigma_{1} \cong 0$.

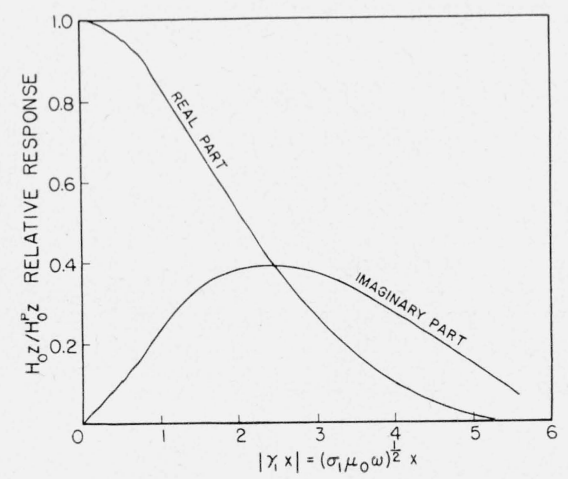

Figure 6. Response of a line source on a homogeneous half-space.

\section{Line Source Over a Thin Layer}

The general solution for $M$ layer can also be specialized conveniently to the case of a line source at a height $h$ over a thin sheet of thickness $h_{1}$ and conductivity $\sigma_{1}$. The following substitutions in eq (48) are made: $\mu_{m}=\mu_{0}, M=2, \gamma_{2}=\gamma_{0}$ where it is supposed that the medium above and below the sheet is homogeneous. The electric field for $z<0$ is given by

$$
E_{0}=-\frac{i \mu_{0} \omega I}{2 \pi}\left\{K_{0}\left(\gamma_{0} r_{1}\right)-K_{0}\left(\gamma_{0} r_{2}\right)+\int_{0}^{\infty} \frac{2 e^{u_{0}(z-h)} \cos \lambda x d \lambda}{u_{e}+u_{0}}\right\}
$$


where

$$
\begin{gathered}
u_{e}=u_{1} \frac{u_{0}+u_{1} \tanh u_{1} h_{1}}{u_{1}+u_{0} \tanh u_{1} h_{1}} \\
r_{1}^{2}=(z+h)^{2}+x^{2} \text { and } r_{2}^{2}=(z-h)^{2}+x^{2} .
\end{gathered}
$$

For small values of the sheet thickness $h_{1}$ and large values of the conductivity $\sigma$, tanh $u_{1} h_{1} \cong u_{1} h_{1}$ and $\epsilon_{1} \omega / \sigma_{1}<<1$,

and therefore

$$
u_{1}^{2} h_{1}=\left(\lambda^{2}+\gamma_{1}^{2}\right) h_{1} \cong 2 i q,
$$

where $q=\sigma_{1} u_{0} \omega h_{1} / 2$. With these approximations, $u_{e} \cong u_{0}+2 i q$ and then

$$
E_{0} \cong \frac{-i \mu_{0} \omega I}{2 \pi}\left[K_{0}\left(\gamma_{0} r_{1}\right)-K_{0}\left(\gamma_{0} r_{2}\right)+\int_{0}^{\infty} \frac{e^{u_{0}(z-h)} \cos \lambda x d \lambda}{u_{0}+i q}\right]
$$

Using the result appropriate for large $q$,

and noting

$$
\frac{1}{u_{0}+i q}=\frac{1}{i q} \sum_{n=1}^{\infty}\left(\frac{u_{0}}{i q}\right)^{m}(-1)^{m},
$$

$$
u_{0}^{m} e^{u_{0}(z-h)}=\left(\frac{\partial}{\partial z}\right)^{m+1} \frac{e^{u_{0}(z-h)}}{u_{0}},
$$

it follows that, asymptotically, ${ }^{9}$

$$
E_{0} \cong \frac{-i \mu_{0} \omega I}{2 \pi}\left[K_{0}\left(\gamma_{0} r_{1}\right)-K_{0}\left(\gamma_{0} r_{2}\right)+\sum_{m=0}^{\infty} \frac{(-1)^{m}}{(i q)^{m+1}}\left(\frac{\partial}{\partial z}\right)^{m+1} K_{0}\left(\gamma_{0} r_{2}\right)\right],
$$

where use has been made of the relation

valid for $(z-h)<0$.

$$
\int_{0}^{\infty} \frac{e^{u_{0}(z-h)}}{u_{0}} \cos \lambda x d \lambda=K_{0}\left(\gamma_{0} r_{2}\right),
$$

The field $E_{0}$ can thus be developed into an (asymptotic) series of inverse powers of $q r_{2}$ where the coefficients involve derivatives with respect of the modified Bessel functions. Using the relations $K_{0}^{\prime}=-K_{1}$ and $K_{0}^{\prime \prime}=-K_{1}^{\prime}=K_{0}+K_{1} / x$, the $n$th derivative of $K_{0}$ can be expressed in terms of $K_{0}$ and $K_{1}$. The first few terms of the resulting series are given below:

where

$$
E_{0}=\frac{-i \mu_{0} \omega I}{2 \pi}\left[K_{0}\left(\gamma_{0} r_{1}\right)-K_{0}\left(\gamma_{0} r_{2}\right)+T\left(\gamma_{0} r_{2}, q\right)\right]
$$

$$
\begin{aligned}
& T\left(\gamma_{0} r_{2}, q\right) \cong \sum_{m=0}^{\infty}\left(\frac{i}{q}\right)^{m+1}\left(\frac{\partial}{\partial z}\right)^{m}\left[\frac{\gamma_{0}(z-h) K_{1}\left(\gamma_{0} r_{2}\right)}{r_{2}}\right] \\
& \cong \frac{i}{q r_{2}} \gamma_{0}(z-h) K_{1}\left(\gamma_{0} r_{2}\right)+\frac{1}{\left(q r_{2}\right)^{2}}\left[\gamma_{0}^{2}(z-h)^{2} K_{0}\left(\gamma_{0} r_{2}\right)-\gamma_{0} r_{2}\left(1-2 \frac{(z-h)^{2}}{r_{2}^{2}}\right) K_{1}\left(\gamma_{0} r_{2}\right)\right], \\
& \text { +terms containing } 1 /\left(q r_{2}\right)^{3}, \quad 1 /\left(q r_{2}\right)^{4}, \quad \text { etc. },
\end{aligned}
$$

where $q r_{2}=\sigma_{1} \mu_{0} \omega h_{1} r_{2} / 2$ and $z<0$.

It can be readily verified that the field below the thin conducting sheet $(z>h)$ is given by

$$
E_{2}=-\frac{i \mu_{0} \omega I}{2 \pi}\left[T\left(\gamma_{0} r_{1}, q\right)\right] \text { for } z>0 \text {. }
$$

As a check, it can be seen that $E_{0}=E_{2}$ at $z=0$. Expressions for the magnetic field components can be found from Maxwell's equations:

$$
H_{m x}=\frac{1}{i \mu_{0} \omega} \frac{\partial E_{m}}{\partial z} \text { and } H_{m z}=-\frac{1}{i \mu_{0} \omega} \frac{\partial E_{m}}{\partial x} \text { for } m=0,2 \text {. }
$$

\footnotetext{
${ }^{9} \mathrm{~A}$ more rigorous derivation of this series expansion is given in reference [7].
} 
The above expansions developed in inverse powers of $\left(q r_{2}\right)$ are particularly suitable for highly conductive sheets when the source or the observer are not near the sheet. Alternative expansions can be developed for smaller values of $q r_{2}$. These are developed as follows. At low frequencies where $\gamma_{0} r_{1}$ and $\gamma_{0} r_{2}$ are small compared to unity, $\gamma_{0}$ can be set equal to zero, and it readily follows that

$$
E_{0}=\frac{-i \mu_{0} \omega I}{2 \pi}\left[\log \left(r_{2} / r_{1}\right)+T_{0}(\beta)\right]
$$

where

$$
T_{0}(\beta)=\int_{0}^{\infty} \frac{e^{-\lambda \beta} \cos \lambda x}{\lambda+i q} d \lambda \text { and } \beta=h-z \text {. }
$$

Inserting the power-series expansion for $\cos \lambda x, E_{0}$ becomes

$$
T_{0}(\beta)=\sum_{m=0}^{\infty} \frac{(-1)^{m} x^{2 m}}{(2 m !)} \int_{0}^{\infty} \frac{\lambda^{2 m} e^{-\lambda \beta} d \lambda}{\lambda+i q} .
$$

Since

$$
\int_{0}^{\infty} \frac{e^{-\lambda \beta}}{\lambda+i q} d \lambda=-e^{+i \beta q} E i(-i \beta q)
$$

where

$$
E i(-i \beta q)=-\int_{+i \beta q}^{\infty} \frac{e^{-t}}{t} d t
$$

it follows that

$$
T_{0}(\beta)=-\sum_{m=0}^{\infty} \frac{(-1)^{m} x^{2 m}}{(2 m !)} g^{2 m}\left(\frac{\partial}{\partial g}\right)^{2 m}\left[e^{i g} \operatorname{Ei}(-i g)\right]
$$

where $g=\beta q=(h-z) h_{1} \sigma_{1} \mu_{0} \omega / 2$. This is an ascending-series expansion in $g$. When $x=0$, it reduces to

$$
T_{0}(\beta)=-e^{i \beta q} E i(-i \beta q) .
$$

Using the latter result, it follows that the field $E_{0}$ for $z<0$ and $E_{2}$ for $z>0$ in the plane $x=0$ is given by

for $z<0$, and

$$
E_{0}=\frac{-i \mu_{0} \omega I}{2 \pi}\left[\log \frac{|z-h|}{|z+h|}-e^{i|z-h| q} E i(-i|z-h| q)\right]
$$

$$
E_{2}=\frac{+i \mu_{0} \omega I}{2 \pi} e^{i|z+h| q} E i(-i|z+h| q),
$$

for $z>0$.

The exponential integral $\mathrm{Ei}(-i g)$ with imaginary argument can be expressed in terms of the cosine and sine integrals as follows:

where

$$
E i(-i g)=C i(g)+i\left[\frac{\pi}{2}-S i(g)\right]
$$

$$
C i(g)=-\int_{g}^{\infty} \frac{\cos t}{t} d t \text { and } \operatorname{Si}(g)=\int_{0}^{g} \frac{\sin t}{t} d t
$$

are tabulated [8] for real values of $g$.

It might be mentioned that $T_{0}(\beta)$ for $x>0$ can also be expressed in terms of exponential integrals, but then the argument is complex. For example,

$$
\begin{aligned}
T_{0}(\beta) & =\frac{1}{2} \int_{0}^{\infty} \frac{e^{-\lambda(\beta-i x)}}{\lambda+i q} d \lambda+\frac{1}{2} \int_{0}^{\infty} \frac{e^{-\lambda(\beta+i x)}}{\lambda+i q} d \lambda \\
& =-\frac{1}{2} e^{i \beta q}\left[e^{-i q x} E i(-i(\beta-i x) q)+e^{i q x} E i(-i(\beta+i x) q)\right]
\end{aligned}
$$

Unfortunately, the exponontial integral of general complex argument is not well tabulated. 


\section{Radiation Field of the Line Source for Any Number of Layers}

The evaluation of the integrals for the general case of any number of layers is not readily carried out unless certain approximating conditions are introduced. The field $E_{0}$, for the case of the line source and the observer in the interface $(z=h=0)$, is conveniently written

$$
E_{0}=\frac{-i \mu_{0} \omega I}{2 \pi} \int_{0}^{\infty} \frac{\cos \lambda x}{u_{0}+u_{e}} d \lambda
$$

where

$$
u_{e}=u_{1}\left[\frac{\left(Y_{2} / N_{1}\right)+\tanh u_{1} h_{1}}{1+\left(Y_{2} / N_{1}\right) \tanh u_{1} h_{1}}\right]
$$

where $Y_{2}$ is the wave admittance associated with the lower layers and is given by eq (48) et al. When $h_{1}$ becomes sufficiently large, it is seen that $u_{e} \cong u_{1}$ and the fields correspond to a homogeneous half-space.

Now, when the subsurface layers are highly conducting, such that $\left|\gamma_{m}\right|>>\left|\gamma_{0}\right|$, it follows that $u_{m}=\left(\lambda^{2}+\gamma_{m}^{2}\right)^{1 / 2} \cong \gamma_{m}$, since the important values of $\lambda$ are when $\lambda$ is of the order of $\left|\gamma_{0}\right|$. To this approximation

$$
u_{e} \cong\left(\lambda^{2}+\gamma_{e}^{2}\right)^{1 / 2} \cong \gamma_{e},
$$

where $\gamma_{e}$ can be regarded as an effective propagation constant. It is approximately given by

$$
\frac{\gamma_{e}}{\gamma_{1}} \cong\left[\frac{\left(Y_{2} / N_{1}\right)+\tanh u_{1} h_{1}}{1+\left(Y_{2} / N_{1}\right) \tanh u_{1} h_{1}}\right]_{\lambda \simeq 0} .
$$

For a two-layer medium $\left(h_{2} \rightarrow \infty\right), \gamma_{e} \cong \gamma_{1} / Q$, where

$$
Q \cong \frac{\left(\gamma_{1} / \gamma_{2}\right)+\tanh \gamma_{1} h_{1}}{1+\left(\gamma_{1} / \gamma_{2}\right) \tanh \gamma_{1} h_{1}}
$$

which is identical to eq (13).

In analogy to eq (64) for the homogeneous half-space, the field $E_{0}$ for the stratified medium can be written

$$
E_{0} \cong \frac{-i \mu_{0} \omega I \gamma_{0}}{\pi\left(\gamma_{1}^{2}-\gamma_{2}^{2}\right) x} K_{1}\left(\gamma_{0} x\right) Q^{2} \text { for }\left|\gamma_{e} x\right|>>1
$$

The factor $Q^{2}$ can be interpreted as a correction factor to account for the presence of stratification in the half space. A numerical discussion of $Q$ has already been given.

\section{Magnetic Line Source Over a Stratified Medium}

In the previous section, a line source of electric current $I$ over a stratified medium was discussed. The corresponding formal solution for a line source of magnetic current $K$ is obtained by making the transformations: $I \rightarrow K, \quad \sigma_{m}+i \epsilon_{m} \omega \rightarrow i \mu_{m} \omega, i \mu_{m} \omega \rightarrow \sigma_{m}+i \epsilon_{m} \omega, \quad E_{z} \rightarrow H_{z}$, $H_{x}=-E_{x}$, and $H_{y} \rightarrow-E_{y}$. The nature of the resulting integrals in the solution are, however, more complicated than in the electric-current counterpart.

The integral form of the solution can be written, for $z<0$

$$
H_{0}=\frac{-i \omega \epsilon_{0} K}{4 \pi} \int_{-\infty}^{+\infty}\left[e^{ \pm u_{0}(z+h)}+R_{\|}(\lambda) e^{u_{0}(z-h}\right] e^{-i \lambda x} d \lambda
$$

where the $+\operatorname{sign}$ is to be used for $(z+h)<0$ and the $-\operatorname{sign}$ for $(z+h)>0$. The reflection coefficient is now given by

$$
R_{\|}(\lambda)=\frac{K_{0}-Z_{1}}{K_{0}+Z_{1}}
$$


where $K_{0}$ and $Z_{1}$ have their usual meaning. For a homogeneous half-space, for example, $Z_{1}=K_{1}$ and if $\mu_{1}=\mu_{0}$,

$$
R_{\|}(\lambda)=\frac{\gamma_{1}^{2} u_{0}-\gamma_{0}^{2} u_{1}}{\gamma_{1}^{2} u_{0}+\gamma_{0}^{2} u_{1}}
$$

The coefficient $R_{\|}(\lambda)$ can have a pole when $\gamma_{\mathrm{i}}^{2} u_{0}+\gamma_{0}^{2} u_{1}=0$, which is the "resonance" condition discussed previously. This complicates the evaluation of the integral and eliminates the possibility of using the formulas developed for the line-current source in the previous section. (There was no pole in the reflection coefficient $R_{\perp}(\lambda)$ for a homogeneous half-space, because there is no solution of $u_{0}+u_{1}=0$.)

If attention is restricted, however, to the field a large distance from the surface, or if the height $h$ is large, it can be expected that contributions from the pole (which are surface waves) are small compared to the specular component. Then with analogy to eq (57), the field is approximately given by

$$
H_{0} \cong \frac{-i \epsilon_{0} \omega K}{2 \pi}\left\{K_{0}\left[\gamma_{0}\left[x^{2}+(z+h)^{2}\right]\right]+R_{\|}(\bar{\theta}) K_{0}\left[\gamma_{0}\left[x^{2}+(z-h)^{2}\right]\right]\right\},
$$

where $R_{\|}(\bar{\theta})$ is the reflection coefficient for a plane wave (parallel polarization) incident at an angle $\bar{\theta}$ on the interface $(z=0)$ where $\tan \bar{\theta}=x /(z-h)$. This result is only valid in the asymptotic sense, and hence to the same accuracy the formula reads

$$
H_{0} \cong \frac{-i \epsilon \omega K}{2 \pi}\left(\frac{\pi}{2}\right)^{1 / 2}\left[\frac{e^{-\gamma_{0} r_{1}}}{\left(\gamma_{0} r_{1}\right)^{3 / 2}}+R_{\|}(\theta) \frac{e^{-\gamma_{0} r_{2}}}{\left(\gamma_{0} r_{2}\right)^{1 / 2}}\right]
$$

where only the first term of the asymptotic expansion of $K_{0}$ has been retained. As can be verified by the saddle-point method (see next section), this result for $H_{0}$ quoted above is the first term of an asymptotic expansion.

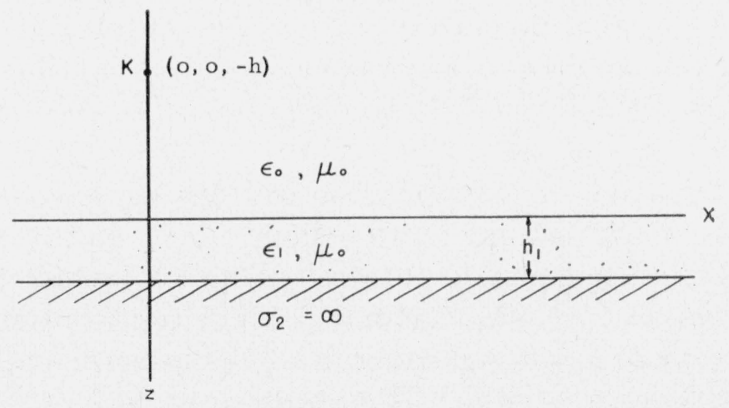

Figure 7. Magnetic line source over a dielectric coated conductor.

\section{Magnetic Line Source Over a Dielectric Coated Conductor}

As mentioned above, the integrals appearing in the solution for parallel polarization are complicated by the existence of poles. This occurs even in the case of a homogeneous halfspace. Another relatively simple situation is when the upper layer is a pure dielectric and the region below is a metallic conductor [9]. The situation is illustrated in figure 7 .

Noting that $Z_{2}=0$, the field for $z<0$ can be expressed by

$$
H_{0}=\frac{-i \epsilon_{0} \omega K}{4 \pi} \int_{-\infty}^{+\infty}\left[e^{ \pm u_{0}(z+h)}+\frac{u_{0}-i k_{0} \Delta}{u_{0}+i k_{0} \Delta} e^{u_{0}(z-h)}\right] e^{-i \lambda x} d \lambda
$$

where

$$
\Delta=\frac{K_{1} \tanh u_{1} h_{1}}{\eta_{0}} \text { and } K_{1}=\frac{u_{1}}{\sigma_{1}+i \omega \epsilon_{1}}
$$


The integral has a pole where $u_{0}+i k_{0} \Delta=0$, and this is denoted by $\lambda_{1}$. Furthermore, there is a branch point where $u_{0}=0$, which is when $\lambda=k_{0}$. Now for small values of $h_{1}$ (i. e., $k_{1} h_{1}<<1$ ) $\Delta$ is also small (compared to one), and therefore

$$
\Delta \cong \frac{K_{1} u_{1} h_{1}}{\eta_{0}} \cong \frac{i \mu_{0} \omega h_{1}}{\eta_{0}}\left(1-\frac{k_{0}^{2}}{k_{1}^{2}}\right)
$$

Therefore, to a first approximation, $\Delta$ does not depend on $\lambda$ the integration variable, and the surface impedance $Z_{1}\left(\cong \eta_{0} \Delta\right)$ is almost purely inductive. The pole is then given by

$$
\lambda_{1} \cong k_{0}\left[1+\left(k_{0} \bar{h}\right)^{2}\right]^{1 / 2} \cong k_{0}\left[1+\left(k_{0} \bar{h}\right)^{2} / 2\right]
$$

where $\bar{h}=h_{1}\left(1-k_{0}^{2} / k_{1}^{2}\right)$

The integral for $H_{0}$ is now in a form where it can be evaluated directly by the modified saddle-point method. A similar integral appears, in the case of a dipolar excitation. For the present purpose, howerer, it is possible to develop the first few terms of the asymptotic expansion in a direct manner.

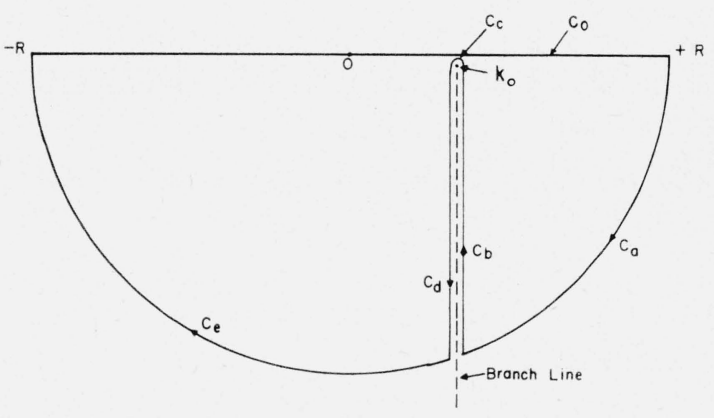

Figure 8. Integration contour in the $\lambda$ plane.

The integration contour is shown in figure 8. The integration in the original integral is along $C_{0}$ from $-\infty$ to $+\infty$. The pole $\lambda_{1}$ and the branch point $k_{0}$ can have a small but vanishing negative imaginary part if the conductivity of the air and the dielectric is finite. The contour $C_{0}$ then passes above $\lambda_{1}$ and $k_{0}$ but passes below $-\lambda_{1}$ and $-k_{0}$.

Following standard practice in contour evaluation of integrals, the contour $C_{0}$ is closed by an infinite semicircle $\left(C_{a}+C_{e}\right)$ in the lower plane, with an indentation along $C_{b}, C_{c}$, and $C_{d}$ to the branch point at $k_{0}$. The integral along $C_{0}$ is then equal to integral along the complete closed contour $C$ minus the individual contributions of $C_{a}, C_{b}, C_{c}, C_{d}$, and $C_{e}$. The contributions from $C_{a}$ and $C_{b}$ vanish as $R \rightarrow \infty$, since the real part of $u_{0}>0$. Furthermore, the integral along the branch lines can be obtained as an inverse series in $x$ (which is asymptotic in nature) by expanding the integrand in a Taylor series and integrating term by term. The integral around the complete contour $C$ is simply $-2 \pi i$ times the residue at the pole $\lambda_{1}$. Finally, it follows that the integral along $C_{0}$ is $-2 \pi i$ times the residue at $\lambda_{1}$ minus the contribution along the branch lines.

The final result reads, for $x>>(h-z)$

$H_{0}=-\epsilon_{0} \omega K\left[\frac{u_{p}}{\lambda_{1}} e^{-u_{p} h} e^{+u_{p} z} e^{-i \lambda_{1} x}+\frac{e^{-i\left(k_{0} x+\pi / 4\right)}}{\sqrt{2 \pi}}\left(\frac{k_{0}}{u_{p}}\right)^{2}\left(1+u_{p} z\right)\left(1-u_{p} h\right) \cdot \frac{1}{\left(k_{0} x\right)^{3 / 2}}\right.$

where

$$
\text { plus terms containing } \left.\frac{1}{\left(k_{0} x\right)^{5 / 2}}, \frac{1}{\left(k_{0} x\right)^{7 / 2}}, \text { etc. }\right] \text {, }
$$

$$
u_{p}=\sqrt{\lambda_{1}^{2}-k_{0}^{2}}=-i k_{0} \Delta
$$

The first term in the brackets is the residue at the pole $\lambda_{1}$, and the succeeding terms are from the branch-line integration. The latter series is asymptotic and is usable if 


$$
\left(\frac{k_{0}}{u_{p}}\right)^{2}<<\left(k_{0} x\right)
$$

which requires that the pole $\lambda_{1}$ is not too near $k_{0}$. Such a condition is violated when the thickness $h_{1}$ of the dielectric coating approaches zero. It is then necessary to apply the modified saddle-point method [10].

\section{Fields of a Vertical Electric Dipole Over a Stratified Half-Space}

In the previous section, the excitation of the fields over a stratified half-space was by a line source. In this case the problem is a two-dimensional one. It is the purpose of this section to mention the extension to the case where the source is a dipole. This problem was formally solved by Sommerfeld in 1909 for the homogeneous half-space.

A vertical electric dipole is to be located at $z=-h$ over the stratified region of $M$ layers. The dipole is to be regarded as a current element of length $d s$ carrying a current $I$.

The situation is illustrated in figure 9 , where it is noted that cylindrical coordinates

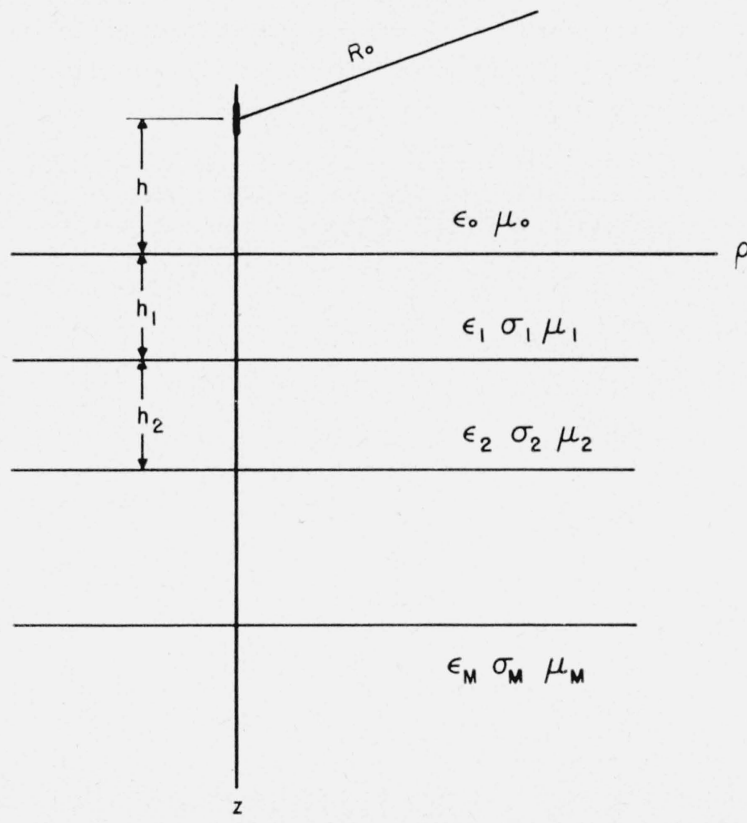

Figure 9. Vertical electric dipole over an M-layered half-space.

$(\rho, \phi, z)$ are employed with the $z$-axis pointing downward as usual. The primary influence in the upper region $(z<0)$ can be expressed in terms of a Hertz vector, which has only a $z$ component $\pi_{z}^{p}$, which hereafter is denoted $\psi^{p}$. Furthermore,

where

$$
\psi^{p}=\frac{I d s}{4 \pi i \omega \epsilon_{0}} \frac{e^{-i k_{0} R_{0}}}{R_{0}},
$$

$$
R_{0}=\left[\rho^{2}+(z+h)^{2}\right]^{1 / 2} .
$$

It is now assumed that the total fields can be expressed everywhere in terms of a Hertz vector, which also has only a $z$ component, denoted $\psi_{m}$. (The justification for doing this is attained by verifying that the final solution satisfies all the boundary conditions and behaves properly at the source and at infinity.) For the $m$ th layer, $\psi_{m}$ satisfies

$$
\left(\nabla^{2}-\gamma_{m}^{2}\right) \psi_{m}=0
$$

except at the source where $\psi_{0} \rightarrow \psi^{p}$, for $R_{0} \rightarrow 0$. This suggests writing

$$
\psi_{0}=\psi^{p}+\psi_{0}^{\prime},
$$


where $\psi_{0}^{\prime}$ is the "secondary influence". Since

then

$$
\left(\nabla^{2}-\gamma_{0}^{2}\right) \psi^{p}=0
$$

$$
\left(\nabla^{2}-\gamma_{0}^{2}\right) \psi^{s}=0
$$

In cylindrical coordinates and for azimuthal symmetry, the equation for $\psi_{m}$ becomes

$$
\left(\frac{1}{\rho} \frac{\partial}{\partial \rho} \rho \frac{\partial}{\partial \rho}+\frac{\partial^{2}}{\partial z^{2}}-\gamma_{m}^{2}\right) \psi_{m}=0
$$

It can be readily seen that solutions of this equation are made up of linear combinations of

$$
e^{ \pm u_{m} z} J_{0}(\lambda \rho)
$$

where, as usual, $u_{m}=\left(\lambda^{2}+\gamma_{m}^{2}\right)^{1 / 2}$, and $\gamma_{m}^{2}=\left(i \sigma_{m} \mu_{m} \omega-\epsilon_{m} \mu_{m} \omega^{2}\right)$, for $m=0,1,2 \ldots$. Again by convention, the real part of $u_{m}$ is to be taken positively. Since the resulting solution is to be finite on the axis $\rho=0$ when $z \neq-h$, the $Y_{0}(\lambda \rho)$ Bessel function can be rejected because it behaves as $\log \rho$ for small values of $\rho$. The $J_{0}(\lambda \rho)$ Bessel function on the other hand, is finite at $\rho=0$.

As in the previous solution for the line source, the parameter $\lambda$ can take any value (with a few exceptions). The general solution for $\psi_{m}$ is then written in the form

$$
\psi_{m}=\int_{0}^{\infty}\left[a_{m}(\lambda) e^{-u_{m} z}+b_{m}(\lambda) e^{u_{m}{ }^{2}}\right] J_{0}(\lambda \rho) d \lambda
$$

for $m=1,2 \ldots, M-1$. In region (0), for $z>0$

$$
\psi_{0}=\psi_{p}+\int_{0}^{\infty} b_{0}^{\prime}(\lambda) e^{u_{0} z} J_{0}(\lambda \rho) d \lambda
$$

and in region $(M)$, for $z>z_{M-1}$

$$
\psi_{M}=\int_{0}^{\infty} a_{M}(\lambda) e^{-u_{m}^{2}} d \lambda
$$

To express $\psi^{p}$ as an integral of the appropriate type, use is made of the Fourier-Bessel transforms, which read

$$
f(\rho)=\int_{0}^{\infty} g(\lambda) J_{0}(\lambda \rho) \lambda d \lambda
$$

and

$$
g(\lambda)=\int_{0}^{\infty} f(\rho) J_{0}(\lambda \rho) \rho d \rho
$$

subject to the conditions that the integrals exist. Letting $f(\rho)=e^{-i k_{0} \rho} / \rho$,

$$
g(\lambda)=\int_{0}^{\infty} e^{-i k_{0} \rho} J_{0}(\lambda \rho) d \rho
$$

This integral is a standard type [5] and is given by

and therefore

$$
g(\lambda)=\frac{1}{\left(\lambda^{2}-k_{0}^{2}\right)^{1 / 2}}=\frac{1}{u_{0}},
$$

$$
\frac{e^{-i k_{0} \rho}}{\rho}=\int_{0}^{\infty} \frac{J_{0}(\lambda \rho)}{\left(\lambda^{2}-k_{0}^{2}\right)^{1 / 2}} \lambda d \lambda=\left.\frac{e^{-i k_{0} R_{0}}}{R_{0}}\right|_{z=-h} .
$$


To generalize the preceding to any other value of $z$, the integrand must contain the factor $e^{ \pm u_{0}(z+h)}$ because $e^{-i k_{0} R_{0}} / R$ is a solution of the wave equation and necessarily becomes $e^{-i k_{0} \rho} / \rho$ as $z \rightarrow-h$. Consequently,

$$
\frac{e^{-i k_{0} R_{0}}}{R_{0}}=\int_{0}^{\infty} \frac{J_{0}(\lambda \rho) e^{ \pm u_{0}(z+h)}}{u_{0}} \lambda d \lambda
$$

where the sign of the exponent is chosen so that the integral converges.

(i. e., + for $(z+h)<0$ and - for $(z+h)>0)$.

The $z$ component of the Hertz vector for the upper region $(z<0)$ can then be written

where

$$
\psi_{0}=\int_{0}^{\infty}\left[a_{0} \frac{e^{ \pm(z+h) u_{0}}}{u_{0}}+b(\lambda) \frac{e^{u_{0}(z-h)}}{u_{0}}\right] J_{0}(\lambda \rho) \lambda d \lambda,
$$

$$
a_{0}=\frac{I d s}{4 \pi i \omega \epsilon_{0}} \text { and } b(\lambda)
$$

is to be determined from the boundary conditions. Now

and

$$
E_{m p}=\frac{\partial^{2} \psi_{m}}{\partial \rho \partial z}, \quad E_{m \phi}=0, \quad E_{m z}=\left(-\gamma_{m}^{2}+\frac{\partial^{2}}{\partial z^{2}}\right) \psi_{m}
$$

$$
H_{m \rho}=0, \quad H_{m \phi}=\frac{-\gamma_{m}^{2}}{i \mu_{m} \omega} \frac{\partial \psi_{m}}{\partial \rho}, \quad H_{m x}=0
$$

and since $E_{m \rho}$ and $H_{m \phi}$ are continuous at the interfaces $z_{m}(m=0$ to $M-1)$, it follows that $\partial \psi_{m} / \partial z$ and $\left(\sigma_{m}+i \omega \epsilon_{m}\right) \psi_{m}$ are also continuous at the same interfaces. This leads to $2(M-1)$ equations to solve for the $2(M-1)$ unknown coefficients. It readily follows that

where

$$
\frac{b_{0}(\lambda)}{a_{0}}=R(\lambda)=\frac{K_{0}(\lambda)-Z_{1}(\lambda)}{K_{0}(\lambda)+Z_{1}(\lambda)}
$$

$$
Z_{m}(\lambda)=K_{m}(\lambda) \cdot \frac{Z_{m+1}(\lambda)+K_{m}(\lambda) \tanh u_{m} h_{m}}{K_{m}(\lambda)+Z_{m+1}(\lambda) \tanh u_{m} h_{m}}
$$

The algebraic form of $R(\lambda)$ is identical to eq (5) for the plane-wave reflection coefficient for parallel incidence. It is thus clear that the field of a dipole over a stratified half-space can be regarded as a spectrum of plane waves whose angle $\theta$ of incidence and reflection is related to the variable $\lambda$ by $\lambda=k_{0} \sin \theta$. In this case, the wave normals generate a family of cones coaxial with the z-axis.

Equations (127) and (128) in conjunction with eq (124) constitute the complete formal solution. Approximate evaluations [10] can be carried out by using the same approach as in the previous sections or by a modified saddle-point method [11]. In view of the similarity with the line-source excitation, it does not seem justified to discuss this in detail, and the essential results will simply be quoted.

As has been demonstrated, $Z_{1}(\lambda)$ for a highly conducting half-space or even for a dielectric coated conductor, is mainly determined by the electrical properties of the layers. In other words, $R(\lambda)$ can be replaced by

$$
R(\lambda)=\frac{u_{0}-i k_{0} \Delta}{u_{0}+i k_{0} \Delta}
$$

where $\Delta=Z_{1} / \eta_{0}=Z_{1} / 120 \pi$ is assumed to be constant. The form of $\psi_{0}$ is then

$$
\psi_{0}=\frac{I d s}{4 \pi i \omega \epsilon_{0}} \int_{0}^{\infty}\left[e^{ \pm u_{0}(z+h)}+\frac{u_{0}-i k_{0} \Delta}{u_{0}+i k_{0} \Delta} e^{u_{0}(z-h)}\right] J_{0}(\lambda \rho) d \lambda .
$$


This can be rewritten as follows:

where

$$
\psi_{0}=\frac{I d s}{4 \pi i \omega \epsilon_{0}}\left[\frac{e^{-i k_{0} R_{0}}}{R_{0}}+\frac{e^{-i k_{0} R_{1}}}{R^{1}}-2 P\right]
$$

and

$$
R_{0}=\left[\rho^{2}+(z+h)^{2}\right]^{1 / 2}, \quad R_{1}=\left[\rho^{2}+(z-h)^{2}\right]^{1 / 2},
$$

$$
P=\int_{0}^{\infty} \frac{\left(i k_{0} \lambda \Delta\right) e^{-u_{0}(h-z)}}{\left(u_{0}+i k_{0} \Delta\right) u_{0}} J_{0}(\lambda \rho) d \lambda .
$$

This integral has been previously evaluated $[10,11]$. Subject to $|\Delta|<<1$, it is given by

where

$$
P \cong i\left(\pi p_{\epsilon}\right)^{1 / 2} e^{-w} \operatorname{erfc}\left(i w^{1 / 2}\right) \frac{e^{-i k_{0} R_{1}}}{R_{1}}
$$

$$
\begin{gathered}
w=p_{e}\left(1+\frac{h-z}{\Delta R_{1}}\right)^{2}, \\
p_{e}=\frac{-i k_{0} R_{1}}{2} \Delta^{2}=\left|p_{e}\right| e^{i b},
\end{gathered}
$$

and

$$
\operatorname{erfc}\left(i w^{1 / 2}\right)=\frac{2}{\sqrt{\pi}} \int_{i w^{1 / 2}}^{\infty} e^{-x^{2}} d x
$$

The expression for $P$ can also be written

where

$$
P=\frac{p_{e}}{w}[1-F(w)] \frac{e^{-i k_{0} R_{1}}}{R_{1}},
$$

$$
F(w)=1-i(\pi w)^{1 / 2} e^{-w} \operatorname{erfc}\left(i w^{1 / 2}\right) .
$$

It is noted that when $z=h=0$,

$$
\psi_{0}=\frac{I d s}{2 \pi i \omega \epsilon_{0}} \frac{e^{-i k_{c} \rho}}{\rho} F\left(p_{e}\right),
$$

and the vertical electric field is given by

$$
E_{o z} \cong \frac{i \mu \omega I d s}{2 \pi \rho} e^{-i k \rho 0} F\left(p_{e}\right) \quad \text { for } k_{0} \rho>>1 .
$$

The function $F\left(p_{e}\right)$ can be regarded as the correction to the field of a dipole on the surface of a perfectly conducting plane. For $|p|<<1$, it approaches unity. $F\left(p_{e}\right)$ has the same functional form as the ground-wave attenuation of Norton [6] who presented numerical values for the case where (in the present notation) $b$ is in the quadrant $0^{\circ}$ to $-180^{\circ}$. For a homogeneous ground $\Delta$ has a phase angle in the range $0^{\circ}$ (for a perfect dielectric) to $45^{\circ}$ (for a good conductor). The corresponding values of $b$ are $-90^{\circ}$ and $0^{\circ}$, respectively. For a stratified ground, however, the phase angle of $\Delta$ may be outside this range. In the particular case of a two-layer conducting: half-space, the parameter $p_{e}$ can be written

$$
p_{e}=p_{1} Q^{2}, \quad \text { where } p_{1}=\frac{-i k_{0} \rho}{2}\left(\eta_{1} / \eta_{0}\right)^{2},
$$

and where

$$
Q=|Q| e^{i q} \cong \frac{\left(\gamma_{1} / \gamma_{2}\right)+\tanh \gamma_{1} h_{1}}{1+\left(\gamma_{1} / \gamma_{2}\right) \tanh \gamma_{1} h_{1}}
$$

in terms of the propagation constants $\gamma_{1}$ and $\gamma_{2}$ of the upper and lower layers and the thickness $h_{1}$ of the upper layer. This function $?$ is discussed in section 2. From figure $3 \mathrm{~b}$ it is seen that 


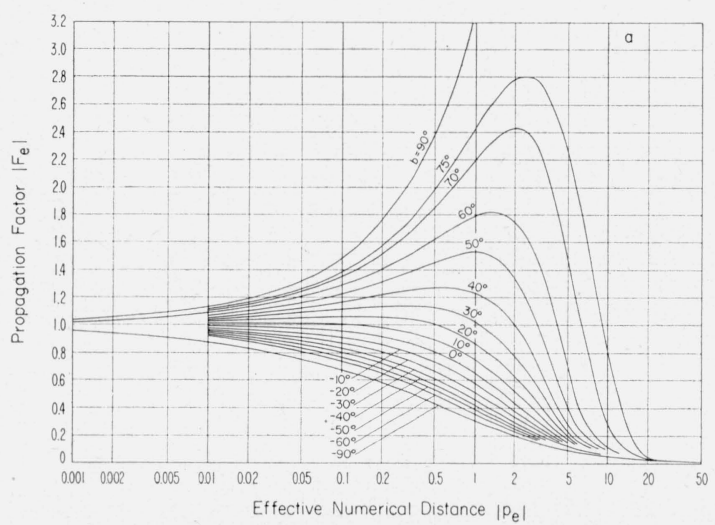

Figure 10a. Amplitude of propagation factor for an impedance boundary.

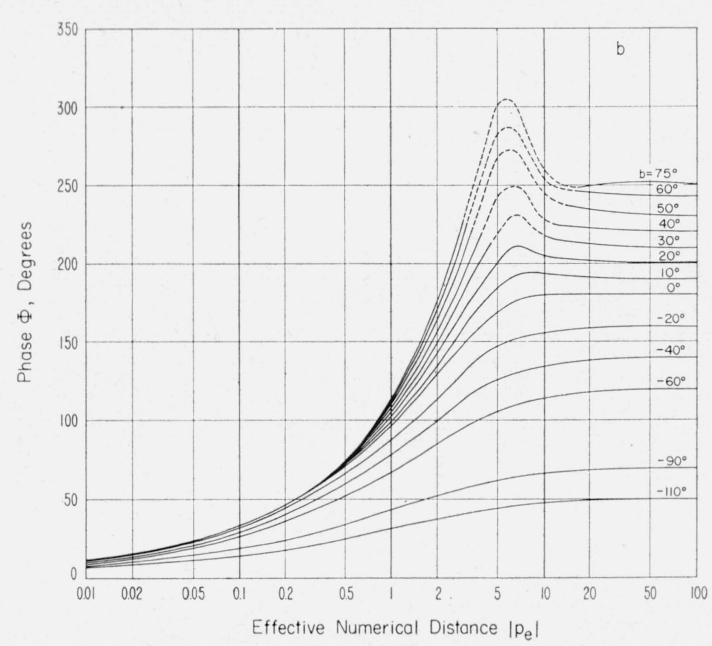

Figure 10b. Phase of the propagation factor for an impedance boundary.

for a highly conducting substratum $q$ can become positive. Consequently, the phase angle $b$ can exceed 0 and would approach $90^{\circ}$ for $\left|\gamma_{2} / \gamma_{1}\right| \rightarrow \infty$, and $\gamma_{1} h_{1}<<1$.

In analogy to the terminology introduced by Sommerfeld for the homogeneous half-space, $p_{e}$ is here described as an effective numerical distance. The propagation factor $F_{e}=\left|F_{e}\right| e^{-i \Phi}$, is shown plotted in figures 10a and $10 \mathrm{~b}$, as a function of $\left|p_{e}\right|$ for a range of $b$ values. The curves for $b<0$ correspond to those computed by Norton, and it is interesting to note that for this region, $\left|F_{e}\right|$ never exceeds unity. On the other hand, when $b>0,\left|F_{e}\right|$ may exceed unity, and for $b=90^{\circ}$ this effect is most pronounced. Apparently, in the case where $b$ is positive, the energy is being guided to some extent along the surface. This effeet can be seen in the asymptotic development of $F\left(p_{e}\right)$, which, for $\left|p_{e}\right|>>1$, reads

when $-2 \pi<b<0$, and

$$
F\left(p_{e}\right) \simeq-\frac{1}{2 p_{e}}-\frac{1 \cdot 3}{\left(2 p_{e}\right)^{2}}-\frac{1 \cdot 3 \cdot 5}{\left(2 p_{e}\right)^{3}}-\frac{1 \cdot 3 \cdot 5 \cdot 7}{\left(2 p_{e}\right)^{4}}-\cdots
$$

when $2 \pi>b>0$.

$$
F\left(p_{e}\right) \simeq-2 i \sqrt{\pi p_{e}} e^{-p_{e}}-\frac{1}{2 p_{e}}-\frac{1 \cdot 3}{\left(2 p_{e}\right)^{2}}-\frac{1 \cdot 3 \cdot 5}{\left(2 p_{e}\right)^{3}}-\cdots
$$

The term $-2 i \sqrt{\pi p_{e}} e^{-p_{e}}$ has all the characteristics of a surface wave. It is not present in the asymptotic development when $b$ is negative. At $b=0,2 \pi$, or $-2 \pi, p_{e}$ is real, and this term vanishes asymptotically. The trapped surface wave is most predominant when $b=90^{\circ}$, which corresponds to $\Delta$ or $Z_{1}$ being positive imaginary. For example, if the surface is coated by a thin dielectric film of thickness $h_{1}$ with a dielectric constant $\epsilon_{1}$,

$$
Z_{1} \cong i \mu \omega h_{1}\left[1-\epsilon_{0} / \epsilon_{1}\right] \cong i X_{1}
$$

which is purely imaginary. Then, since

$$
p_{e}=\frac{-i k_{0} \rho}{2}\left(Z_{1} / \eta_{0}\right)^{2}
$$

$p_{e}=\left|p_{e}\right| e^{i \pi / 2}$, or $b=\pi / 2$ radians $=90^{\circ}$. The attenuation factor now has the asymptotic development

$$
F\left(p_{e}\right) \simeq-2 e^{i 3 \pi / 4} \sqrt{\pi\left|p_{e}\right|} e^{-i\left|p_{e}\right|}+\frac{i}{2\left|p_{e}\right|}+\frac{3}{4\left|p_{e}\right|^{2}}-\frac{15 i}{8\left|p_{e}\right|^{3}} \cdots
$$


where

and the vertical electric field is

$$
\left|p_{e}\right|=\frac{k_{0} \rho}{2} \frac{X^{2}}{\eta_{0}^{2}}
$$

$$
E_{0 z} \cong \frac{i \mu \omega I d s}{2 \pi \rho} e^{-i k_{0} \rho} F\left(p_{e}\right),
$$

which shows that the (trapped) surface wave component varies as

$$
\frac{1}{\sqrt{k_{0} \rho}} \epsilon^{-i k_{0}\left(1+\frac{X^{2}}{2 \eta_{0}^{2}}\right) \rho}
$$

which has the characteristics of a cylindrical wave traveling in the positive $\rho$ with a phase velocity

times that of light.

$$
\left(1+\frac{X^{2}}{2 \eta_{0}^{2}}\right)^{-1}
$$

The (trapped) surface wave is not excited when $b$ is negative, and then the distant electric field varies asymptotically as

$$
\frac{1}{\left(k_{0} \rho\right)^{2}} e^{-i k_{0} \rho}
$$

which has a phase velocity equal to that of light.

When the source dipole and the observer are both raised above the surface (i.e., $h>0$ and $-z>0$ ), the field in the upper half-space can be expressed conveniently in the asymptotic sense as the sum of three partial fields in the manner

$$
\psi_{0} \cong\left[\psi_{a}+\psi_{b}+\epsilon \psi_{s}\right] \frac{I d s}{4 \pi i \omega \epsilon_{0}},
$$

where $\epsilon=0$ for $b<0$, and $\epsilon=1$ for $b>0$. An asymptotic development shows that

$$
\begin{gathered}
\psi_{a} \cong \frac{e^{-i k R_{0}}}{R_{0}}+\frac{C-\Delta}{C+\Delta} \frac{e^{-i k R_{1}}}{R_{1}}, \\
\psi_{b}=-\left\{\frac{1}{p(1+C / \Delta)^{3}}+\frac{1 \cdot 3}{2 p^{2}(1+C / \Delta)^{5}}+\frac{1 \cdot 3 \cdot 5}{4 p^{3}(1+C / \Delta)^{7}}+\ldots\right\} \frac{e^{-i k R_{1}}}{R_{1}}
\end{gathered}
$$

and

$$
\psi_{s} \cong-\frac{2 \Delta}{\Delta+C}\left[2 i \sqrt{\pi w} e^{-w}\right] \frac{e^{-i k R_{1}}}{R_{1}},
$$

where

and $\Delta=Z_{1} / \eta_{0}$.

$$
C=\frac{h-z}{R_{1}}=\frac{h+|z|}{R_{1}}, k=2 \pi / \text { wavelength }=k_{0},
$$
$\left.C^{2}\right) \psi_{0}$.

In terms of $\psi_{0}$, the vertical electric field components for $k_{0} \rho>>1$ is given by $E_{0 z} \cong k_{0}^{2}(1-$

In the above, $\psi_{a}$ can be identified as a geometrical optical term being just the primary field $e^{-i k R_{0}} / \mathrm{R}_{0}$ and a specularly reflected component $e^{-i k R_{1}} / R_{1}$ modified by a reflection coefficient $(C-\Delta) /(C+\Delta) . \quad \psi_{b}$ is an asymptotic expansion containing terms varying as $R_{1}^{-2}, R_{1}{ }^{-3}$, etc. $\psi_{s}$, which is only present for positive $b$ values, represents a (trapped) surface wave and has a phase velocity less than that of light. When $b$ is negative there is no (trapped) surface wave present.

Sometimes $\psi_{a}$ itself is called a space wave and $\psi_{b}$ a surface wave. This usage would correspond to that of Norton [3]. This would be an obvious designation when the half-space is homogeneous because $\psi_{s}$ is zero, and there is no trapped surface wave excited.

Equations (143), (144), and (145) enable one to compute the field in the upper halfspace $(z<0)$ for an electric dipole located at $z=-h$ in terms of the ratio $\Delta$ of the surface im- 
pedance $Z_{1}$ to that of free space $\eta_{0}$. As a first approximation, $Z_{1}$ could be replaced by $Z_{1}(\lambda)$ for $\lambda=0$, which would be the normal surface impedance. Clearly, a better value would be $Z_{1}\left(\lambda_{s}\right)$, where $\lambda_{s}$ is the saddle point of the integral in eq (130) or (132). This would mean that

$$
\Delta=\frac{Z_{1}\left(\lambda_{s}\right)}{\eta_{0}}
$$

where $\lambda_{s}=k_{0} S$, where $S=\rho / R_{1}=\sqrt{1-C^{2}}$. For a homogeneous half-space, with $\mu_{1}=\mu_{0}$,

$$
\begin{aligned}
\Delta & =\frac{K_{1}\left(\lambda_{s}\right)}{\eta_{0}}=\frac{u_{1}\left(\lambda_{s}\right)}{\left(\sigma_{1}+i \omega \epsilon_{1}\right) \eta_{0}} \\
& =\frac{\eta_{1}}{\eta_{0}}\left(1-\frac{\eta_{1}^{2}}{\eta_{0}^{2}} S^{2}\right)^{1 / 2} \cdot
\end{aligned}
$$

The coefficient $(C-\Delta) /(C+\Delta)$ in eq (143) now can be identified as the Fresnel reflection coefficient for a plane wave with parallel polarization incident at angle $\theta$. The equations are now in complete agreement with the results of Norton [6] for a vertical electric dipole over a homogeneous conducting ground.

The corresponding treatment for a vertical magnetic dipole located at $z=-h$ over the $M$ layered half-space is almost identical to the above. The quantity $\psi_{0}$ is then to be identified with the $z$ component of the magnetic Hertz vector, and then $\Delta$ is to be defined by $\Delta=\eta_{0} Y_{1}$, where $Y_{1}$ is the surface admittance at the interface $z=0$.

\section{Appendix}

\subsection{Effect of a Transition Region on Reflection}

The reflection problems considered above were entirely concerned with layered media. Each layer was assumed to be homogeneous and bounded by a sharp interface.

In certain instances the dielectric constant or magnetic permeability can be regarded to vary in a simple analytical fashion. In such cases, it is sometimes possible to obtain a solution of Maxwell's equations in terms of known functions. The reflection coefficient for a medium in which the dielectric constant may be expressed as a power of one coordinate has been considered in great detail by Försterling [12]. The hyperbolic transition has been considered by Rydbeck [14].

In this appendix, a treatment of the linear transition between two lossless dielectrics will be given. Actually, this is a special case of Försterling's model, but the mathematics is somewhat simpler.

The situation is illustrated in figure 11. A plane wave is incident at angle $\theta$ from the air

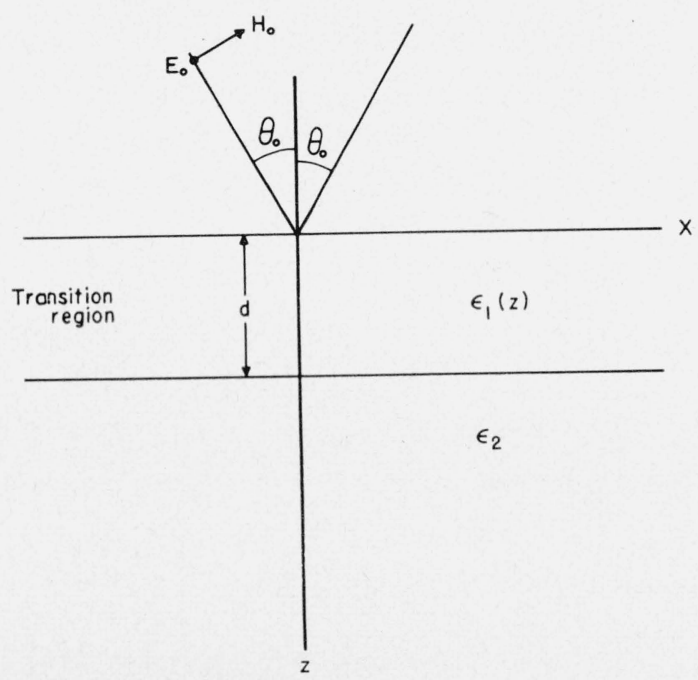

Figure 11. Illustrating a plane-wave incident on a linear transition region. 
to an interface at $z=0$. The electric vector is perpendicular to the plane of incidence. The dielectric constant between $z=0$ and $z=d$ is a function of the coordinate $z$ and denoted $\epsilon_{1}(z)$. The region below, $z>d$, has a dielectric constant $\epsilon_{2}$, which does not vary with $z$. The magnetic permeability of the whole space is taken as $\mu_{0}$ and assumed to be constant.

The electric field, which has only a $y$ component, can then be written

$$
E_{0}=\left[e^{-i k_{0} z \cos \theta}+R e^{i k_{0} z \cos \theta_{0}}\right] e^{-i k_{0} x \sin \theta_{0}} \text { for } z<0,
$$

and

$$
E_{2}=T e^{-i k_{2} z \operatorname{cs} \theta_{2}} e^{-i k_{0} x \sin \theta_{0}} . \text { for } z>d,
$$

where $\theta_{2}$ is related to $\theta_{1}$ by $k_{2} \sin \theta_{2}=k_{0} \sin \theta_{0}$. The constants $R$ and $T$ are to be determined by the boundary conditions at $z=0$ and $z=d$.

Within the transition layer, the field components satisfy Maxwell's equations

$$
\begin{gathered}
\frac{\partial E_{1}}{\partial z}=i \mu_{0} \omega H_{1 x}, \\
i \mu_{0} \omega H_{1 z}=i k_{0} \sin \theta_{0} E_{1},
\end{gathered}
$$

and

$$
\frac{\partial H_{1 x}}{\partial z}=i\left[\epsilon_{1}(z)-\epsilon_{0} \sin ^{2} \theta_{0}\right] \omega E_{1},
$$

where $E_{1}$ is the $y$ component of the electric field in the transition region. For a linear transition,

$$
\epsilon_{1}(z)=\epsilon_{0}(1+\beta z)
$$

where $\beta=\left(\epsilon_{2}-\epsilon_{0}\right) / \epsilon_{0} d$. A new variable $W$ is now introduced, defined by

$$
W=W_{0}\left(1+\frac{\beta z}{\cos ^{2} \theta}\right)^{3 / 2} \text { and } W_{0}=\frac{2\left(\epsilon_{1}^{1 / 2} \cos ^{3} \theta\right)}{3 \beta} \text {. }
$$

Equation (151a) and (151b) can then be written

$$
\epsilon^{1 / 2} \frac{d E_{1}}{d W}=i \mu_{0} \omega H_{1 x} \quad \text { and } \quad \frac{d H_{1 x}}{d W}=i \omega \epsilon^{1 / 2} E_{1}
$$

where $\boldsymbol{\epsilon}=\epsilon_{1}(z)-\epsilon_{0} \sin ^{2} \theta$.

These equations are combined to give

$$
\left(\frac{d^{2}}{d W^{2}}+\frac{1}{2 \epsilon} \frac{d \epsilon}{d W} \frac{d}{d W}+\omega^{2} \mu_{0}\right) E_{1}=0 .
$$

But, since $\epsilon=$ const. $\times W^{2 / 3}$,

Furthermore, if the substitution

$$
\frac{1}{2 \epsilon} \frac{d \epsilon}{d W}=\frac{1}{3 W}
$$

$$
E_{1}=W^{1 / 3} \psi
$$

is made, $\psi$ satisfies the equation

$$
W^{2} \frac{d^{2} \psi}{d W^{2}}+W \frac{d \psi}{d W}+\left(\mu \omega^{2} W^{2}-\frac{1}{3^{2}}\right) \psi=0
$$

which is Bessel's equation of order $1 / 3$. Independent solutions are $J_{1 / 3}\left(\omega \mu^{1 / 2} W\right)$ and $J_{-1 / 3}$ $\left(\omega \mu^{1 / 2} W\right)$. 
The general solution for $E_{1}$ is then written

$$
E_{1}=\left(W / W_{0}\right)^{1 / 3}\left[A J_{1 / 3}\left(\omega \mu^{1 / 2} W\right)+B J_{-1 / 3}\left(\omega \mu^{1 / 2} W\right)\right] \times \exp \left(-i k_{0} x \sin \theta_{0}\right),
$$

where $A$ and $B$ are constants. The magnetic-field components are obtained from

$$
H_{1 x}=\frac{1}{i \mu_{0} \omega} \frac{\partial E_{1}}{\partial z} \text { and } H_{1 z}=\frac{k_{0} \sin \theta}{\mu_{0} \omega} E_{1}
$$

and need not be written out.

The four unknown constants $R, T, A$, and $B$ are obtained by matching the tangential fields at $z=0$ and $z=d$. That is,

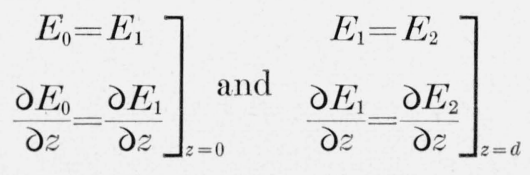

Omitting algebraic details, the expression for the reflection coefficient can be finally written

$$
R=\frac{1-G}{1+G},
$$

where

$$
G=\frac{\left(a_{1} p_{0}+q_{0} b_{1}\right)-i\left(p_{0} q_{1}-q_{0} p_{1}\right)}{\left(b_{0} q_{1}+a_{0} p_{1}\right)-i\left(a_{0} b_{1}-a_{1} b_{0}\right)}
$$

where

with

$$
\begin{aligned}
& a_{0}=J_{1 / 3}\left(x_{0}\right), \quad p_{0}=J_{23}\left(x_{0}\right), \\
& b_{0}=J_{-1 / 3}\left(x_{0}\right), \quad q_{0}=J_{-2 / 3}\left(x_{0}\right), \\
& a_{1}=J_{1 / 3}\left(x_{1}\right), \quad p_{1}=J_{2 / 3}\left(x_{1}\right), \\
& b_{1}=J_{-1 / 3}\left(x_{1}\right), \quad q_{1}=J_{-23}\left(x_{1}\right),
\end{aligned}
$$

$$
x_{0}=\frac{\alpha}{N^{2}-1} \cdot \frac{4 \pi}{3}, \quad x_{1}=\frac{\alpha N^{3}}{N^{2}-1} \cdot \frac{4 \pi}{3},
$$

and

$$
\begin{aligned}
\alpha & =\frac{k_{0} d}{2 \pi} \cos \theta_{0}=\frac{d}{\lambda_{0}} \cdot \cos \theta_{0}, \\
N & =\frac{\left(\epsilon_{2} / \epsilon_{0}-\sin ^{2} \theta_{0}\right)^{1 / 2}}{\cos \theta} .
\end{aligned}
$$

Employing tables of fractional order Bessel functions [15], the magnitude of the reflection coefficient $|R|$, is plotted in figure 12 as a function of $\alpha$ or $\left(d / \lambda_{0}\right) \cos \theta_{0}$. The abscissa is proportional to the thickness of the transition region. Two values of $N$ are chosen, 3 and 5 .

It is immediately apparent that the effect of the transition region is to reduce the reflection coefficient. For small values of $\left(d \cos \theta_{0} / \lambda_{0}\right)$, the reflection is governed almost entirely by the (normalized) relative refractive index $N$. In fact, as $d \rightarrow 0$, it is readily seen that

$$
R=\frac{1-N}{1+N}=\frac{\cos \theta_{0}-\left(\epsilon_{2} / \epsilon_{1}-\sin ^{2} \theta_{0}\right)^{1 / 2}}{\cos \theta+\left(\epsilon_{2} / \epsilon_{1}-\sin ^{2} \theta_{0}\right)^{1 / 2}}
$$

which is the classical Fresnel formula for perpendicular incidence. As seen in figure $12|R|=$ 0.5 for $N=3$, and $d=0 ;|R|=0.666$ for $N=5$; and $d=0$.

In the language of transmission line theory, the transition layer is acting as a matching 


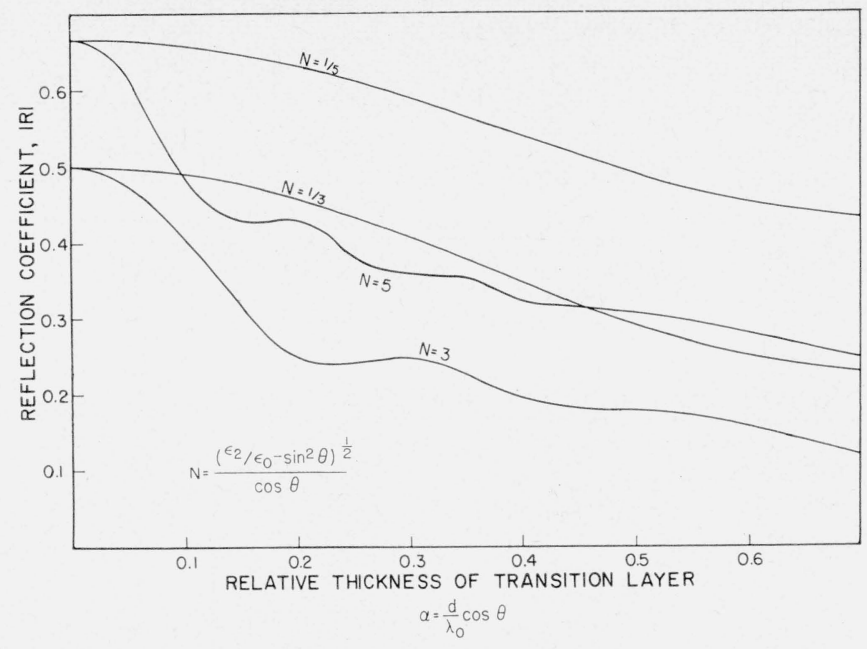

FIGURE 12. Reflection coefficient for a linear transition layer.

section. Its function is to match the impedance in the air region $(z<0)$ to that of the lower region $(z>d)$.

The simplicity of the above analysis is due primarily to the choice of the polarization of the incident wave. In the corresponding problem for parallel incidence, the resulting equations for the magnetic field component in the transition layer are not expressible in terms of Bessel functions.

I thank K. A. Norton for advice and H. E. Bussey and A. G. McNish for constructive criticism of the manuscript.

\section{References}

[1] J. C. Zenneck, Ann. Phys. 23, 846 (1907).

[2] A. N. Sommerfeld, The propagation of waves in wireless telegraphy, Ann. Phys. 4, 665 (1909).

[3] K. A. Norton, Propagation of radio waves over a plane earth, Nature 135, 954 (June 1935); K. A. Norton, The propagation of radio waves over the earth and in the upper atmosphere, pt. I, Proc, IRE 24, 1367 (Oct. 1936); pt. II, Proe, IRE 25, 1203 (Sept. 1937). K. A. Norton, The calculation of groundwave field intensity over a finitely conducting sperical earth, Proc. IRE 29, 623 (Dec. 1941).

[4] J. R. Wait, Propagation of radio waves over a stratified ground, Geophysics 18, 416 (April 1953).

[5a] S. S. Atwood, Surface wave propagation over a coated plane conductor, J. Appl. Phys. 22, 504 (1951).

[5b] G. Goubau, On the excitation of surface waves, Proc. Inst. Radio Engrs. 40, 865 (1952).

[6] G. Campbell and R. Foster, Fourier integrals for practical application (Van Nostrand Co. Ltd., New York, N. Y., 1948).

[7] J. R. Wait, Fields of a line current source over a stratified conductor, App]. Sci. Research, sec. B 3 (1953).

[8] E. Jahnke and F. Emde, Tables of functions, 4th ed. (Dover Publications, New York, N. Y., 1945).

[9] A. L. Cullen, The excitation of plane surface waves, Inst. Elect. Engrs. Monograph No. 93 (Feb. 1954).

[10] J. R. Wait, Excitation of surface waves on conducting, stratified, dielectric-clad, and corrugated surfaces, J. Research NBS 59, 365 (1957) RP2807.

[11] J. R. Wait, Radiation from a vertical dipole over a stratified ground, pt. I, Trans. IRE, AP-1, 9 (July 1953) and pt. II, Trans. IRE AP-2, 144 (Oct. 1954).

[12] Karl Forsterling, On reflection in a non-homogeneous medium, Ann. Phys. 11, 1 (1931) and 8, (new series) $129(1950)$.

[13] P. S. Epstein, Reflection of waves in an inhomogeneous absorbing medium, Proc. Nat. Acad. Sci. Am. 16, 627 (1930).

[14] O. Rydbeck, The reflection of electromagnetic waves from a parabolic ionized layer, Phil. Mag. 34, 342 (1943).

[15] Tables of bessel functions of fractional order (Columbia University Press, 1949).

Boulder, Colo., February 1958. 